\title{
Small black hole constituents and horizontal symmetry
}

\author{
Anna Ceresole, ${ }^{a}$ Sergio Ferrara, ${ }^{b, c, d}$ Alessio Marrani ${ }^{b, c}$ and Armen Yeranyan ${ }^{c, e}$ \\ a INFN, Sezione di Torino, \\ Via Pietro Giuria 1, I-10125 Torino, Italy \\ ${ }^{b}$ Physics Department, Theory Unit, CERN, \\ CH -1211, Geneva 23, Switzerland \\ ${ }^{c}$ INFN - Laboratori Nazionali di Frascati, \\ Via Enrico Fermi 40, I-00044 Frascati, Italy \\ ${ }^{d}$ Department of Physics and Astronomy, \\ University of California, Los Angeles, CA 90095-1547, U.S.A. \\ ${ }^{e}$ Department of Physics, Yerevan State University, \\ Alex Manoogian St. 1, Yerevan, 0025, Armenia \\ E-mail: ceresole@to.infn.it, sergio.ferrara@cern.ch, \\ alessio.marrani@cern.ch, ayeran@lnf.infn.it
}

ABSTRACT: By exploiting the role of the horizontal symmetry $S L_{h}(2, \mathbb{R})$, we extend the analysis and classification of two-centered extremal black hole charge configurations to the case of "small" single-centered constituents. These latter are seen to decrease the number of independent horizontal-invariant polynomials from four to one, depending on the rank of the charge orbit supporting each of the two centers. Within $U$-duality groups of type $E_{7}$, both reducible and irreducible symmetric supergravity models in four space-time dimensions are considered, thus encompassing $\mathcal{N}=2$ and $\mathcal{N}=8$ theories.

Keywords: Black Holes in String Theory, Black Holes, Supergravity Models

ARXIV EPRINT: 1104.4652 


\section{Contents}

1 Introduction 1

$\begin{array}{lll}2 & U \text {-invariant constraints on } \mathcal{Q} & 7\end{array}$

$\begin{array}{llr}3 & \text { Two-centered relations } & 9\end{array}$

4 Reducible models $\quad 12$

$\begin{array}{lll}4.1 & \text { Generic reducible models } & 13\end{array}$

$\begin{array}{lll}4.2 & \text { The } s t^{2} \text { model } & 15\end{array}$

$\begin{array}{llr}5 & \text { Irreducible models } & \mathbf{1 7}\end{array}$

$\begin{array}{ll}5.1 \text { Generic irreducible models } & 17\end{array}$

$\begin{array}{ll}5.2 \text { The } t^{3} \text { model } & 19\end{array}$

6 A comment on bound states with negative discriminant 21

\section{Introduction}

Multi-centered black holes (BH) are a fascinating subject, dealing with several aspects of quantum gravity, when this theory is defined through supergravity, and its high-energy, fundamental completions, namely superstrings and $M$-theory. The discovery of the split attractor flow and of walls of marginal stability [1-3] and the corresponding issue of microstate BH counting [4]-[6], have been some remarkable achievements in this field, also characterized by some puzzling and yet not fully understood features, such as anti-marginal stability and entropy enigmas (see also [7]-[19]; for studies on $\mathcal{N}>2$, see [20]-[25]). Earlier studies on composite (super)gravity solutions and marginal stability, were done in [28-32], while [33] provides a recent review on wall-crossing formulæ.

This paper continues the investigation of geometric aspects of $\mathrm{BH}$ physics, by exploiting the (classical) duality symmetries of the underlying supergravities [34], which are the continuum limit of the U-duality [36] governing the non-perturbative string dynamics, in the context of two-centered $\mathrm{BH}$ solutions. The ultimate aim is to show that different aspects of multi-centered $\mathrm{BH}$ dynamics are encoded into different values of (and constraints among) certain multi-centered duality polynomial invariants. These duality invariants characterize some multi-centered charge orbits, which generalize the electric-magnetic charge orbits encoding all the main features of single-centered BH solutions, such as the BekensteinHawking entropy [37, 38], the ADM mass [39] and the BPS (supersymmetry-preserving) properties [40-43].

The single-centered orbits [40] are known to provide a stratification of the space of the irrep. $\mathbf{R}$ of the $d=4$ classical $U$-duality group $G_{4}$ of the $\mathbf{f}$-dimensional electric-magnetic 
charge vector $\mathcal{Q} \equiv\left(p^{\Lambda}, q_{\Lambda}\right)(\Lambda=1, \ldots, \mathbf{f} / 2)$. Analogously, the $p$-centered orbits are expected to stratify the space $\mathbf{R}_{1} \times \cdots \times \mathbf{R}_{p}$, for $p$ BH constituents, with electro-magnetic fluxes given by

$$
\mathcal{Q}_{a}^{M} \equiv\left(p_{a}^{\Lambda}, q_{a \Lambda}\right) \quad a=1, \ldots, p, \quad, \quad M=1, \ldots, \mathbf{f} .
$$

Interestingly, it has been recently uncovered $[44,45]$ that a $p(\geqslant 2)$-centered $\mathrm{BH}$ solution in $d=4$ space-time dimensions enjoys an "horizontal" symmetry $S L_{h}(p, \mathbb{R})$ among the centers of the $\mathrm{BH}$ constituents. As a consequence of this symmetry, further invariant polynomials in the charges beside the usual $U$-duality invariants acquire an important role, and they provide a tool to achieve a finer classification of the allowed two-centered configurations. ${ }^{1}$

As commented in [45], the analysis can be performed for a generic number $p$ of centers, but, due to the structure of the split flow in multi-center (super)gravity solutions [1-4], the case $p=2$ is already fully illustrative, at least regarding marginal stability. It is then natural to explore the charge orbits for the horizontal doublet $\left(\mathcal{Q}_{1}, \mathcal{Q}_{2}\right)$, and to classify the allowed quantum numbers for the extremal $\mathrm{BH}$ two-centered compounds by suitable invariant constraints among horizontal invariant-polynomials.

For $p=2$, the simplest example of such "horizontal invariants" is provided by the symplectic product of two charge vectors $[1,45]$

$$
\mathcal{W} \equiv\left\langle\mathcal{Q}_{1}, \mathcal{Q}_{2}\right\rangle=\frac{1}{2} \mathcal{Q}_{a}^{M} \mathcal{Q}_{b}^{N} \mathbb{C}_{M N} \epsilon^{a b},
$$

where $a, b=1,2, \mathbb{C}_{M N}$ is the symplectic metric

$$
\mathbb{C}_{M N} \equiv\left(\begin{array}{cc}
0 & -\mathbb{I} \\
\mathbb{I} & 0
\end{array}\right),
$$

and $\epsilon$ is the usual $S L(2, \mathbb{R}) \sim S p(2, \mathbb{R})$ rank-2 invariant tensor. By considering $\left(\mathcal{Q}_{1}, \mathcal{Q}_{2}\right)$ as a doublet $\mathcal{Q}_{a}$ (spin $s=1 / 2$, fundamental irrep. 2) of $S L_{h}(2, \mathbb{R})$ [44], it is evident from (1.2) that $\mathcal{W}$ is manifestly invariant under both the $U$-duality and the horizontal symmetry. It is known that $\mathcal{W}$ enters the description of many physical properties of the two-centered $\mathrm{BH}$ compound states, such as the equilibrium distance between the two centers, the intrinsic overall (orbital) angular momentum, and the marginal stability condition [1,3]. Indeed, a crucial feature of two-centered BH physics is that their physical properties turn out to depend not only on $\mathcal{Q}_{1}+\mathcal{Q}_{2}$, as for the BPS ADM mass [47], but also on other combination of charges, such as the symplectic product (1.2). Another important instance is the entropy at the (split) horizon, which is the sum of the entropies of the two single-centered $\mathrm{BH}$ constituents [3]:

$$
\frac{S_{1+2}}{\pi}=\sqrt{\left|\mathcal{I}_{4}\left(\mathcal{Q}_{1}\right)\right|}+\sqrt{\left|\mathcal{I}_{4}\left(\mathcal{Q}_{2}\right)\right|}
$$

where $\mathcal{I}_{4}$ is the unique quartic invariant polynomial of the irrep. $\mathbf{R}$ of the $U$-duality group $G_{4}$,

$$
\mathcal{I}_{4}(\mathcal{Q})=\frac{1}{2} \mathbb{K}_{M N P Q} \mathcal{Q}^{M} \mathcal{Q}^{N} \mathcal{Q}^{P} \mathcal{Q}^{Q}
$$

\footnotetext{
${ }^{1}$ The horizontal symmetry has recently been investigated within the fascinating connections with Quantum Information Theory in [46]
} 
and $\mathbb{K}_{M N P Q}$ is the so-called $K$-tensor [48]. As a natural two-centered generalization of the quartic invariant $I_{4}(\mathcal{Q})$, for $p=2$ one considers the contraction of the $K$-tensor with four a priori different charge vectors, which gives rise to the symmetric $\mathbf{I}_{a b c d}$ tensor [44, 45], sitting in the spin $s=2$ irrep. 5 of $S L_{h}(2, \mathbb{R})$ :

$$
\mathbf{I}_{a b c d} \equiv \frac{1}{2} \mathbb{K}_{M N P Q} \mathcal{Q}_{a}^{M} \mathcal{Q}_{b}^{N} \mathcal{Q}_{c}^{P} \mathcal{Q}_{d}^{Q}
$$

If $G_{4}$ is a simple group, one can define the symmetric object in the horizontal indices [45]

$$
T_{(a b)}^{\alpha}=t_{M N}^{\alpha} \mathcal{Q}_{a}^{M} \mathcal{Q}_{b}^{N}
$$

where $t^{\alpha}{ }_{M N}\left(\alpha=1, \ldots, \mathbf{d} \equiv \operatorname{dim}_{\mathbb{R}}\left(\mathbf{A d j}\left(G_{4}\right)\right)\right)$ is the symplectic representation of the generators of the Lie algebra $\mathfrak{g}_{4}$ of $G_{4}$. Using these tensors, one has

$$
\mathbf{I}_{(a b c d)}=-\frac{1}{6 \tau} t_{(M N}^{\alpha} t_{\alpha \mid P Q)} \mathcal{Q}_{a}^{M} \mathcal{Q}_{b}^{N} \mathcal{Q}_{c}^{P} \mathcal{Q}_{d}^{Q}=-\frac{1}{6 \tau} T_{(a b}^{\alpha} T_{\alpha \mid c d)}
$$

where $\tau$ is a model-dependent parameter [48]:

$$
\tau \equiv \frac{2 \mathbf{d}}{\mathbf{f}(\mathbf{f}+1)}
$$

A similar structure arises also when $G_{4}$ is a semisimple group factorized as $\operatorname{SL}(2, \mathbb{R}) \times$ $\mathrm{SO}(m, n)$, characterizing the reducible symmetric models listed in table 1 . Indeed, within the so-called Calabi-Vesentini basis $[49,50]$ symplectic frame, one can define the $\mathbb{T}$ tensor [44]

$$
T_{(a b)[\Lambda \Sigma]}=p_{\Lambda(a} q_{b) \Sigma}-q_{\Lambda(a} p_{b) \Sigma}=\left(\begin{array}{ll}
\mathbb{T}_{11} & \mathbb{T}_{12} \\
\mathbb{T}_{12} & \mathbb{T}_{22}
\end{array}\right)_{\Lambda \Sigma},
$$

where indices are raised with the the pseudo-Euclidean $\mathrm{SO}(m, n)$ metric $\eta_{\Lambda \Sigma}$. This is a symmetric object in the horizontal indices, whose components are the triplet of $\mathbb{T}$-tensors of the reducible models (called $\mathbb{T}_{1}, \mathbb{T}_{2}, \mathbb{T}_{12}$ in [44]). They constitute a two-centered generalization of the product $T_{\Lambda \Sigma}=p_{\Lambda} q_{\Sigma}-q_{\Lambda} p_{\Sigma}$, which appears in the single-centered fourth order invariant written as [51-54]

$$
\mathcal{I}_{4}=\frac{1}{2} T_{\Lambda \Sigma} T_{\Gamma \Delta} \eta^{\Lambda \Gamma} \eta^{\Sigma \Delta}=-\frac{1}{2} \operatorname{Tr}_{\eta}\left(\mathbb{T}^{2}\right)=p^{2} q^{2}-(p \cdot q)^{2},
$$

where " $\operatorname{Tr}_{\eta}$ " denotes the $\eta$-trace, namely the trace in which the indices are contracted with $\eta$. For two centers, in reducible models it generalizes to

$$
\mathbf{I}_{a b c d}=\frac{1}{2} T_{(a b \mid \Lambda \Sigma} T_{\mid c d) \Gamma \Delta} \eta^{\Lambda \Gamma} \eta^{\Sigma \Delta}=-\frac{1}{2} \operatorname{Tr}_{\eta}\left(\mathbb{T}_{(a b} \mathbb{T}_{c d)}\right) .
$$

The study of two-center extremal $\mathrm{BH}$ charge orbits associated to a generic horizontal doublet $\mathcal{Q}_{1}, \mathcal{Q}_{2}$ has been initiated in $[44,45]$. It was found that in $d=4$ supergravity theories with symmetric scalar manifolds and for generic charge vectors for each of the two centers, the dimension of a complete basis of $U$-duality invariant-polynomials is seven, and it includes both the horizontal singlet $\mathcal{W}$ and the quintet $\mathbf{I}_{a b c d}$. The minimum number 
of invariant polynomials has been shown to decrease to four if only polynomials invariant under both $U$-duality and horizontal symmetry are taken into account. The dimension of this $G_{4} \times S L_{h}(2, \mathbb{R})$ invariant basis further reduces in some specific cases, for instance, for some rank-2 and rank-1 symmetric scalar manifolds pertaining to the so-called $s t^{2}$ and $t^{3}, \mathcal{N}=2, d=4$ models, and for theories whose BH charge irrep. admits a quadratic invariant polynomial $\left|\mathcal{I}_{2}\right|=\sqrt{\left|\mathcal{I}_{4}\right|}$, namely, $\mathcal{N}=2$ minimally coupled [64] and for $\mathcal{N}=3$ supergravity theories [55], which will not be dealt with in this paper. Interestingly, the "pure" $\mathcal{N}=4, d=4$ supergravity, despite having a scalar manifold $\frac{S L(2, \mathbb{R})}{U(1)}$ of rank 1 , has a complete basis formed by seven duality invariants [44].

The present investigation completes the previous analysis in that it determines the restrictions and constraints on such invariants when at least one of the two centers is occupied by a "small" black hole, having $\mathcal{I}_{4}(\mathcal{Q})=0$. Small black holes, corresponding to horizonless solutions in Einstein two-derivatives (super)gravity, have zero entropy and they don't show an attractor behavior [40]. However, they are an interesting sector of the BH spectrum that has been recently examined in $[42,56]$, in particular regarding the different supersymmetry features of the allowed orbits.

Large black hole charge orbits, with $\mathcal{I}_{4} \neq 0$ are described by a minimum of four charges, and in this sense the corresponding orbits are "rank four". More precisely, the rank of the orbit (or of the charge vector $\mathcal{Q}$ spanning it) is here the minimal number of charges which compose an orbit representative. Mathematically speaking, this defines the rank of $\mathcal{Q}$ as element of the associated Freudenthal triple system [57, 58]. However, large orbits can become "lightlike" when they satisfy the condition $\mathcal{I}_{4}=0$, and their rank reduces to three. If a further differential constraint $\partial \mathcal{I}_{4} / \partial \mathcal{Q}=0$ is imposed, the rank further reduces to two (critical orbits), and it becomes one for doubly-critical orbits, having a suitable projection of $\partial^{2} \mathcal{I}_{4} /(\partial \mathcal{Q})^{2}$ vanishing [59]. In section 2 we shall revisit and add new results to the manifestly $U$-invariant constraints defining the rank, ranging from 4 to 1 , of the single-centered charge vector $\mathcal{Q}$, which gives rise to the stratification of the representation space of the $U$-duality group. When combining two centers, these constraints will clearly reflect in a number of combinations for the orbits of the compound system, which will be thoroughly investigated.

In section 3 we shall deal with relations and properties of the invariant polynomials characterizing a two-centered (extremal) $\mathrm{BH}$ compound, which admit a natural interpretation and classification in terms of the horizontal symmetry group $S L_{h}(2, \mathbb{R})$.

Then, the results of sections 2 and 3 are used to perform a detailed analysis of all possible two-centered (extremal) BH charge configurations, by considering all possible combinations of the ranks of the charge vectors $\mathcal{Q}_{1}$ and $\mathcal{Q}_{2}$ pertaining to the two single-centered BH constituents.

This analysis is carried out for all supergravity theories (with symmetric scalar manifold) whose $d=4 U$-duality group $G_{4}$ is a "group of type $E_{7}$ ", namely a group with a symplectic representation $\mathbf{R}$ admitting a completely symmetric rank- 4 invariant structure 


\begin{tabular}{|c|c|c|c|}
\hline $\mathcal{N}$ & $\frac{G_{4}}{m c s\left(G_{4}\right)}$ & rank & $\begin{array}{c}J_{3} \\
\text { reducible }\end{array}$ \\
\hline 2 & $\frac{S L_{v}(2, \mathbb{R})}{U(1)} \times \frac{S O(2, n)}{S O(2) \times S O(n)}, n \in \mathbb{N}$ & $1+\min (2, n)$ & $\mathbb{R} \oplus \boldsymbol{\Gamma}_{1, n-1}$ \\
\hline 4 & $\frac{S L_{v}(2, \mathbb{R})}{U(1)} \times \frac{S O(6, n)}{S O(6) \times S O(n)}, n \in \mathbb{N} \cup\{0\}$ & $1+\min (6, n)$ & $\mathbb{R} \oplus \boldsymbol{\Gamma}_{5, n-1}$ \\
\hline
\end{tabular}

Table 1. Reducible symmetric $d=4$ supergravity models. "mcs" stands for maximal compact subgroup (with symmetric embedding). The rank of the scalar manifold, as well as the related reducible Euclidean rank-3 Jordan algebra $J_{3}$ are also given (for further elucidation, see e.g. [70] and refs. therein). The subscript " $v$ " stands for "vertical", and it has been introduced in order to distinguish the $S$-duality $S L_{v}(2, \mathbb{R})$ group from the horizontal symmetry group $S L_{h}(2, \mathbb{R})$

\begin{tabular}{|c|c|c|c|}
\hline $\mathcal{N}$ & $\frac{G_{4}}{m c s\left(G_{4}\right)}$ & rank & $\begin{array}{c}J_{3} \\
\text { irreducible }\end{array}$ \\
\hline 2 & $\frac{S L_{v}(2, \mathbb{R})}{U(1)}$ & 1 & $\mathbb{R}$ \\
$\left(t^{3}\right.$ model $)$ & $\frac{S p(6, \mathbb{R})}{U(3)}$ & 3 & $J_{3}^{\mathbb{R}}$ \\
\hline 2 & $\frac{S U(3,3)}{S(\mathrm{U}(3) \times U(3))}$ & 3 & $J_{3}^{\mathbb{C}}$ \\
\hline 2 & $\frac{S O^{*}(12)}{U(6)}$ & 3 & $J_{3}^{\mathbb{H}}$ \\
\hline $2 \stackrel{\text { "twin" }}{\leftrightarrow} 6$ & $\frac{E_{7(-25)}}{E_{6(-78)} \times U(1)}$ & 3 & $J_{3}^{\mathbb{Q}}$ \\
\hline 2 & $\frac{S U(1,5)}{U(5)}$ & 1 & $M_{1,2}(\mathbb{O})$ \\
\hline 5 & $\frac{E_{7(7)}}{S U(8)}$ & 7 & $J_{3}^{\mathbb{Q}}$ \\
\hline 8 & &
\end{tabular}

Table 2. Irreducible symmetric $d=4$ supergravity models. $\mathcal{N}=2$ magical quaternionic MaxwellEinstein supergravity and $\mathcal{N}=6$ "pure" supergravity are "twin", namely they share the same bosonic sector [71-75]. $M_{1,2}(\mathbb{O})$ is the Jordan triple system (not upliftable to $d=5$ ) generated by $2 \times 1$ matrices over $\mathbb{O}[76,77]$. Note that, with the exception of the reducible - but triality symmetric - stu model [52, 78], irreducible models are all ones for which the treatment of [48] holds (see e.g. table 1 therein)

q such that the invariant polynomial $\mathcal{I}_{4}$ can be defined $\operatorname{as}^{2}[61]$

$$
\begin{aligned}
\mathcal{I}_{4}(\mathcal{Q}) & \left.\equiv \frac{1}{2} \mathbf{q}\left(\mathbf{q}_{1}, \mathbf{q}_{2}, \mathbf{q}_{3}, \mathbf{q}_{4}\right)\right|_{\mathbf{q}_{1}=\mathbf{q}_{2}=\mathbf{q}_{3}=\mathbf{q}_{4} \equiv \mathcal{Q}} \\
& \equiv \frac{1}{2} \mathbb{K}_{M N P Q} \mathcal{Q}^{M} \mathcal{Q}^{N} \mathcal{Q}^{P} \mathcal{Q}^{Q} .
\end{aligned}
$$

It is also worth recalling that the "group of type $E_{7}$ " $G_{4}$ is a symmetry group of Jordan

\footnotetext{
${ }^{2}$ The normalization of $\mathbf{q}$ used here is the same as in [60], and thus it differs by a factor 2 with respect to the one adopted e.g. in $[48,61]$ and [45]. The same holds e.g. for eq. (3.1) further below.
} 
algebra-related structures, namely:

$$
G_{4} \sim \operatorname{Aut}\left(\mathfrak{M}\left(J_{3}\right)\right) \sim \operatorname{Conf}\left(J_{3}\right),
$$

where $\operatorname{Aut}\left(\mathfrak{M}\left(J_{3}\right)\right)$ is the automorphism group of the vector space $\mathfrak{M}\left(J_{3}\right) \equiv \mathbb{R} \oplus \mathbb{R} \oplus$ $J_{3} \oplus J_{3}$ constructed over the Euclidean rank-3 Jordan algebra $J_{3}$, whose conformal group is $\operatorname{Conf}\left(J_{3}\right)$ (see e.g. [62,63] for recent reviews and lists of refs.). On the other hand, the $G_{4}$ 's of minimally coupled $\mathcal{N}=2$, and $\mathcal{N}=3$ [65] supergravities, omitted in this investigation, do not enjoy an interpretation in terms of (rank-3 Euclidean) Jordan algebras.

The groups of type $E_{7} G_{4}$ 's (which are $U$-duality groups of supergravity theories in $d=4$ space-time dimensions with symmetric scalar manifolds) may be grouped into two classes, depending on whether $G_{4}$ is a semisimple Lie group, or it is a simple Lie group itself. The former case, analyzed in section 4, corresponds to the reducible symmetric models, whose scalar manifolds ${ }^{3}$ are grouped into two infinite sequences, reported in table 1. The first $(n=1)$ element of the $\mathcal{N}=2$ sequence in table 1 , namely the so-called $s t^{2}$ model, is non-generic, and it deserves a separate treatment, given in section 4.2. The corresponding two-centered (extremal) $\mathrm{BH}$ charge orbits with both $\mathcal{Q}_{1}$ and $\mathcal{Q}_{2}$ "large" have been studied in [44]. Generally, the number of independent $G_{4}$-invariant is seven, and a complete basis can be taken to be [44]

$$
\mathcal{W}, \mathcal{X}, \mathbf{I}_{a b c d}
$$

where the quartic polynomial $\mathcal{X}$ is defined by eq. (3.20).

The latter case, analyzed in section 5 , corresponds to the irreducible symmetric models, namely to the so-called $\mathcal{N}=2 t^{3}$ model, to the $\mathcal{N}=2$ magical Maxwell-Einstein supergravity theories as well as to $\mathcal{N}=5,6,8$ "pure" supergravities, whose scalar manifolds are reported in table 2 . The $t^{3}$ model is non-generic and it deserves a separate treatment, given in section 5.2 .

The two-centered (extremal) BH "large" charge orbits of generic irreducible symmetric models and of $t^{3}$ model have been studied respectively in [45] and in [44]. Generically, the number of independent $G_{4}$-invariant is seven, and a complete basis can be taken to be [45]

$$
\mathcal{W}, \mathbf{I}_{a b c d}, \mathbf{I}_{6},
$$

where the sextic polynomial $\mathbf{I}_{6}$ is defined by eq. (3.15). The main difference between the sets (1.15) and (1.16) is that $\mathcal{X}=0$ (3.21) in irreducible cases, where also the constraint (4.8) of degree twelve in the charges does not hold. It will be emphasised in section 3 that the existence of $\mathcal{X}$ in reducible models can be traced back to the semi-simple nature of the $U$-duality group $G_{4}$, giving rise to two independent quartic polynomials $\mathbf{I}^{\prime}$ and $\mathbf{I}^{\prime \prime}$ with vanishing horizontal helicity, and related to $\mathbf{I}_{0}$ by eq. (3.9).

The outcome of this analysis is that the number of independent duality- and horizontalinvariant polynomials is decreased when one or both charge vectors are "small", since the

\footnotetext{
${ }^{3}$ In matter coupled theories, we consider vector multiplets' scalar manifolds; for instance, this is the case for all models of table 1 , with the exception of "pure" $\mathcal{N}=4$ supergravity.
} 
vanishing of the quartic form (1.5) for one center or both gives rise to various polynomial relations among otherwise independent invariants. For example, as yielded by the analysis of sections 4 and 5 , if at least one of the two charge vectors $\mathcal{Q}_{1}$ and $\mathcal{Q}_{2}$ is doubly-critical (i.e. rank 1 , see section 2 ; in the $\mathcal{N}=8$ language, this is $\frac{1}{2}$-BPS), then only one independent horizontal invariant, say the symplectic product $\mathcal{W}(1.2)$, exists. This is one of the few examples known in the literature [3].

Notice that the results concerning the $s t^{2}$ and $t^{3}$ models are consistent with the "stu $\rightarrow$ $s t^{2} \rightarrow t^{3}$ reduction" (see e.g. the discussion in [44, 66, 67], and the recent analysis in [46]).

Finally, in section 6 we make some comments on some "small + small" two-centered charge configurations corresponding to $\mathcal{I}_{4}\left(\mathcal{Q}_{1}+\mathcal{Q}_{2}\right)<0$, i.e. to a $\mathrm{BH}$ compound that, regarded as a single-centered solution, is a "large" non-BPS (extremal) $\mathrm{BH}$, making contact with recent literature, such as [11] and [68].

\section{$2 U$-invariant constraints on $\mathcal{Q}$}

We start by giving a résumé (with original results) of the $U$-invariant constraints defining the charge orbits of a single-center (extremal) BH, namely of the $G_{4}$-invariant conditions defining the rank of the dyonic charge vector $\mathcal{Q} \in \mathbf{R}$ as an element of the corresponding Freudenthal triple system (FTS) (see [57, 58], and refs. therein); as mentioned above, $G_{4}$ is the $d=4 U$-duality group "of type $E_{7}$ " [61], and $\mathbf{R}$ is its relevant $\mathrm{BH}$ charge irrep. The symplectic indices $M=1, \ldots, \mathbf{f}\left(\mathbf{f} \equiv \operatorname{dim}_{\mathbb{R}} \mathbf{R}\left(G_{4}\right)\right)$ are raised and lowered with the symplectic metric $\mathbb{C}_{M N}$ defined by (1.3). By recalling the definition (1.5) of the unique (quartic) $G_{4^{-}}$ invariant polynomial constructed with $\mathcal{Q} \in \mathbf{R}$, the rank of a non-null $\mathcal{Q}$ as an element of $\operatorname{FTS}\left(G_{4}\right)$ range from four to one, and it is manifestly $G_{4}$-invariantly characterized as follows:

1. $\operatorname{rank}(\mathcal{Q})=4$ : "large" extremal BHs, with non-vanishing area of the event horizon:

$$
\mathcal{I}_{4}(\mathcal{Q}) \gtrless 0 \text {. }
$$

2. $\operatorname{rank}(\mathcal{Q})=3$ : "small" lightlike extremal BHs, with vanishing area of the event horizon:

$$
\begin{aligned}
& \mathcal{I}_{4}(\mathcal{Q})=0 \\
& \frac{\partial \mathcal{I}_{4}}{\partial \mathcal{Q}^{M}} \neq 0 \Leftrightarrow \mathbb{K}_{M N P Q} \mathcal{Q}^{N} \mathcal{Q}^{P} \mathcal{Q}^{Q} \neq 0, \text { at least for some } M .
\end{aligned}
$$

3. $\operatorname{rank}(\mathcal{Q})=2$ : "small" critical extremal BHs:

$$
\begin{aligned}
\frac{\partial \mathcal{I}_{4}}{\partial \mathcal{Q}^{M}} & =0 \Leftrightarrow \mathbb{K}_{M N P Q} \mathcal{Q}^{N} \mathcal{Q}^{P} \mathcal{Q}^{Q}=0, \forall M \\
\left.\frac{\partial^{2} \mathcal{I}_{4}}{\partial \mathcal{Q}^{M} \partial \mathcal{Q}^{N}}\right|_{\operatorname{Adj}\left(G_{4}\right)} & \neq 0, \text { at least for some } M, N
\end{aligned}
$$


4. $\operatorname{rank}(\mathcal{Q})=1$ : "small" doubly-critical extremal BHs [59, 79]:

$$
\left.\frac{\partial^{2} \mathcal{I}_{4}}{\partial \mathcal{Q}^{M} \partial \mathcal{Q}^{N}}\right|_{\mathbf{A d j}\left(G_{4}\right)}=0 \Leftrightarrow\left(3 \mathbb{K}_{M N P Q}+\mathbb{C}_{M P} \mathbb{C}_{N Q}\right) \mathcal{Q}^{P} \mathcal{Q}^{Q}=0, \forall M, N .
$$

Let us consider the doubly-criticality condition (2.6) more in detail. At least for "groups of type $E_{7}$ " [61] which are $U$-duality groups $G_{4}$ of irreducible symmetric models in $d=4$, it holds that

$$
\begin{aligned}
& (\mathbf{R} \times \mathbf{R})_{s}=\mathbf{A d j}+\mathbf{S} \\
& (\mathbf{R} \times \mathbf{R})_{a}=\mathbf{1}+\mathbf{A},
\end{aligned}
$$

where the subscripts " $s$ " and " $a$ " clearly stand for symmetric and skew-symmetric. The presence of the singlet (which is nothing but $\mathbb{C}_{M N}$ defined in (1.3)) in the skew-symmetric part (2.8) characterizes the BH charge irrep. $\mathbf{R}$ to be symplectic. For example, for $G_{4}=E_{7}$ $(\mathbf{R}=\mathbf{5 6}, \mathbf{A d j}=\mathbf{1 3 3})$ one gets (see e.g. [80]; the subscripts "s" and "a" respectively stand for symmetric and antisymmetric)

$$
\begin{aligned}
& (56 \times 56)_{s}=133+1463 \\
& (56 \times 56)_{a}=1+1539 .
\end{aligned}
$$

For such groups, one can construct the projector operator on $\mathbf{A d j}\left(G_{4}\right)$ :

$$
\begin{aligned}
\mathcal{P}_{A B}{ }^{C D} & =\mathcal{P}_{(A B)}^{(C D)} ; \\
\mathcal{P}_{A B}{ }^{C D} \frac{\partial^{2} \mathcal{I}_{4}}{\partial \mathcal{Q}^{C} \partial \mathcal{Q}^{D}} & =\left.\frac{\partial^{2} \mathcal{I}_{4}}{\partial \mathcal{Q}^{A} \partial \mathcal{Q}^{B}}\right|_{\operatorname{Adj}\left(G_{4}\right)} ; \\
\mathcal{P}_{A B}{ }^{C D} \mathcal{P}_{C}{ }_{D}^{E F} \frac{\partial^{2} \mathcal{I}_{4}}{\partial \mathcal{Q}^{E} \partial \mathcal{Q}^{F}} & =\mathcal{P}_{A B}{ }^{E F} \frac{\partial^{2} \mathcal{I}_{4}}{\partial \mathcal{Q}^{E} \partial \mathcal{Q}^{F}},
\end{aligned}
$$

where (recall (2.7))

$$
\begin{aligned}
\frac{\partial^{2} \mathcal{I}_{4}}{\partial \mathcal{Q}^{A} \partial \mathcal{Q}^{B}} & =\left.\frac{\partial^{2} \mathcal{I}_{4}}{\partial \mathcal{Q}^{A} \partial \mathcal{Q}^{B}}\right|_{\mathbf{A d j}\left(G_{4}\right)}+\left.\frac{\partial^{2} \mathcal{I}_{4}}{\partial \mathcal{Q}^{A} \partial \mathcal{Q}^{B}}\right|_{\mathbf{S}\left(G_{4}\right)} ; \\
\left.\frac{\partial^{2} \mathcal{I}_{4}}{\partial \mathcal{Q}^{A} \partial \mathcal{Q}^{B}}\right|_{\mathbf{A d j}\left(G_{4}\right)} & =2(1-\tau)\left(3 \mathbb{K}_{A B C D}+\mathbb{C}_{A C} \mathbb{C}_{B D}\right) \mathcal{Q}^{C} \mathcal{Q}^{D} \\
\left.\frac{\partial^{2} \mathcal{I}_{4}}{\partial \mathcal{Q}^{A} \partial \mathcal{Q}^{B}}\right|_{\mathbf{S}\left(G_{4}\right)} & =2\left[3 \tau \mathbb{K}_{A B C D}+(\tau-1) \mathbb{C}_{A C} \mathbb{C}_{B D}\right] \mathcal{Q}^{C} \mathcal{Q}^{D},
\end{aligned}
$$

where the model-dependent parameter $\tau$ is defined by (1.9). The explicit expression of $\mathcal{P}_{A B}^{C D}$ reads $^{4}(\alpha=1, \ldots, \mathbf{d})$ :

$$
\mathcal{P}_{A B}^{C D}=\tau\left(3 \mathbb{C}^{C E} \mathbb{C}^{D F} \mathbb{K}_{E F A B}+\delta_{(A}^{C} \delta_{B)}^{D}\right)=-t^{\alpha \mid C D} t_{\alpha \mid A B},
$$

\footnotetext{
${ }^{4}$ For related results in terms of a map formulated in the " $4 D / 5 D$ special coordinates" symplectic frame (and thus manifestly covariant under the $d=5 U$-duality group $G_{5}$ ), see e.g. [81, 82].
} 
where the relation [48] (see also [69])

$$
\mathbb{K}_{M N P Q}=-\frac{1}{3 \tau} t_{(M N}^{\alpha} t_{\alpha \mid P Q)}=-\frac{1}{3 \tau}\left[t_{M N}^{\alpha} t_{\alpha \mid P Q}-\tau \mathbb{C}_{M(P} \mathbb{C}_{Q) N}\right]
$$

where

$$
t_{M N}^{\alpha}=t_{(M N)}^{\alpha} ; t_{M N}^{\alpha} \mathbb{C}^{M N}=0
$$

is the symplectic representation of the generators of the Lie algebra $\mathfrak{g}_{4}$ of $G_{4}$. Notice that $\tau<1$ defined in (1.9) is just the ratio of the dimensions of the adjoint Adj and rank-2 symmetric $(\mathbf{R} \times \mathbf{R})_{s}(2.7)$ reps. of $G_{4}$, or equivalently the ratio of upper and lower indices of $t_{M N}^{\alpha}$ 's themselves. It should also be noted that, with respect to the treatment given in [48], the result (2.18) has been supplemented with the relation $\xi=-\frac{1}{3 \tau}$ [45], obtained as a consistency condition within the computations yielding to (2.17).

The result (2.17) is a direct consequence of the fact that $t_{M N}^{\alpha}$ is the projector of $(\mathbf{R} \times \mathbf{R})_{s}$ onto Adj (recall (2.7)). More precisely, it holds that

$$
\mathcal{P}_{A B}{ }^{C D} t_{C D}^{\alpha}=t_{A B}^{\alpha}
$$

where the normalization (see e.g. eq. (2.5) of [48])

$$
t_{F}^{\alpha{ }_{F}} t_{C}^{\beta F}=g^{\alpha \beta}
$$

has been used.

\section{Two-centered relations}

In order to study multi-centered charge configurations, it is worth considering some general relations for $p(\geqslant 2$ )-center (extremal) BHs, which are manifestly covariant under the horizontal symmetry $S L_{h}(p, \mathbb{R})$ introduced in [44]; we will here focus on the case $p=2$.

From [44, 45] and the Introduction, we recall $\mathbf{I}_{a b c d}$ and

$$
\widetilde{\mathcal{Q}}_{M \mid a b c} \equiv \frac{1}{4} \frac{\partial \mathbf{I}_{a b c d}}{\partial \mathcal{Q}_{d}^{M}}=\frac{1}{2} \mathbb{K}_{M N P Q} \mathcal{Q}_{a}^{N} \mathcal{Q}_{b}^{P} \mathcal{Q}_{c}^{Q}=\widetilde{\mathcal{Q}}_{M \mid(a b c)}
$$

respectively sitting in the spin $s=2$ and $s=3 / 2$ of $S L_{h}(2, \mathbb{R})$ (the horizontal indices $a=1,2$ are raised and lowered with $\epsilon^{a b}$, with $\epsilon^{12} \equiv 1$ ). For clarity's sake, we report the explicit expressions of the various components of $\mathbf{I}_{a b c d}$, as well as their relations with the components of $\widetilde{\mathcal{Q}}_{a b c}(3.1)$ (the subscripts " $+2,+1,0,-1,-2$ " denote the horizontal helicity 
of the various components $[44,45])$ :

$$
\begin{aligned}
& \mathbf{I}_{+2} \equiv \mathcal{I}_{4}\left(\mathcal{Q}_{1}\right) \equiv \mathbf{I}_{1111}=\left\langle\widetilde{\mathcal{Q}}_{111}, \mathcal{Q}_{1}\right\rangle ; \\
& \mathbf{I}_{+1} \equiv \mathbf{I}_{1112}=\left\langle\widetilde{\mathcal{Q}}_{111}, \mathcal{Q}_{2}\right\rangle=\left\langle\widetilde{\mathcal{Q}}_{112}, \mathcal{Q}_{1}\right\rangle ; \\
& \mathbf{I}_{0} \equiv \mathbf{I}_{1122}=\left\langle\widetilde{\mathcal{Q}}_{112}, \mathcal{Q}_{2}\right\rangle=\left\langle\widetilde{\mathcal{Q}}_{122}, \mathcal{Q}_{1}\right\rangle ; \\
& \mathbf{I}_{-1} \equiv \mathbf{I}_{1222}=\left\langle\widetilde{\mathcal{Q}}_{122}, \mathcal{Q}_{2}\right\rangle=\left\langle\widetilde{\mathcal{Q}}_{222}, \mathcal{Q}_{1}\right\rangle ; \\
& \mathbf{I}_{-2} \equiv \mathcal{I}_{4}\left(\mathcal{Q}_{2}\right) \equiv \mathbf{I}_{2222}=\left\langle\widetilde{\mathcal{Q}}_{222}, \mathcal{Q}_{2}\right\rangle .
\end{aligned}
$$

Note that in reducible symmetric models, due to the semi-simple nature of $G_{4}$, there are two independent components of $\mathbf{I}_{a b c d}$ with vanishing horizontal helicity, namely $\mathbf{I}^{\prime}$ and $\mathbf{I}^{\prime \prime}[44]$ :

$$
\begin{aligned}
\mathbf{I}^{\prime} & \equiv-\frac{1}{2} \operatorname{Tr}_{\eta}\left(\mathbb{T}_{11} \mathbb{T}_{22}\right) ; \\
\mathbf{I}^{\prime \prime} & \equiv-\frac{1}{2} \operatorname{Tr}_{\eta}\left(\mathbb{T}_{12}^{2}\right),
\end{aligned}
$$

and related to $\mathbf{I}_{0}$ by the relation (4.4) of [44]:

$$
\mathbf{I}_{0}=\frac{1}{3}\left(\mathbf{I}^{\prime}+2 \mathbf{I}^{\prime \prime}\right)
$$

Thus, one can consider the following symplectic product of spin $3 / 2$ horizontal charge tensors:

$$
\left\langle\widetilde{\mathcal{Q}}_{a b c}, \widetilde{\mathcal{Q}}_{d e f}\right\rangle \equiv \widetilde{\mathcal{Q}}_{M \mid a b c} \widetilde{\mathcal{Q}}_{N \mid d e f} \mathbb{C}^{M N}
$$

A priori, $\left\langle\widetilde{\mathcal{Q}}_{a b c}, \widetilde{\mathcal{Q}}_{\text {def }}\right\rangle$ should project onto spin $s=3,2,1,0$ irreps. of $S L_{h}(2, \mathbb{R})$ itself; however, due to the complete symmetry of the $K$-tensor (and to the results of $[48,61]$ ), the projections on spin $s=3$ and 1 do vanish:

$$
\begin{aligned}
& s=3:\left\langle\widetilde{\mathcal{Q}}_{(a b c}, \widetilde{\mathcal{Q}}_{d e f)}\right\rangle=0 \\
& s=2:\left\langle\widetilde{\mathcal{Q}}_{(a b \mid c}, \widetilde{\mathcal{Q}}_{d \mid e f)}\right\rangle \epsilon^{c d}=\frac{2}{3} \mathcal{W} \mathbf{I}_{a b e f} ; \\
& s=1:\left\langle\widetilde{\mathcal{Q}}_{(a \mid b c}, \widetilde{\mathcal{Q}}_{d e \mid f)}\right\rangle \epsilon^{b d} \epsilon^{c e}=0 ; \\
& s=0:\left\langle\widetilde{\mathcal{Q}}_{a b c}, \widetilde{\mathcal{Q}}_{d e f}\right\rangle \epsilon^{a d} \epsilon^{b e} \epsilon^{c f}=8 \mathbf{I}_{6},
\end{aligned}
$$

where the symplectic product $\mathcal{W}$ is defined by (1.2), and in (3.14) the definition of the sextic horizontal polynomial $\mathbf{I}_{6}$ [45] (given by eq. (3.24) of [45]) has been recalled:

$$
\mathbf{I}_{6} \equiv \frac{1}{8}\left\langle\widetilde{\mathcal{Q}}_{a b c}, \widetilde{\mathcal{Q}}_{d e f}\right\rangle \epsilon^{a d} \epsilon^{b e} \epsilon^{c f}=\frac{1}{4}\left\langle\widetilde{\mathcal{Q}}_{111}, \widetilde{\mathcal{Q}}_{222}\right\rangle+\frac{3}{4}\left\langle\widetilde{\mathcal{Q}}_{122}, \widetilde{\mathcal{Q}}_{112}\right\rangle .
$$


The complementary relation to $(3.15)$, namely $\frac{1}{4}\left\langle\widetilde{\mathcal{Q}}_{111}, \widetilde{\mathcal{Q}}_{222}\right\rangle-\frac{3}{4}\left\langle\widetilde{\mathcal{Q}}_{122}, \widetilde{\mathcal{Q}}_{112}\right\rangle$ consistently turns out to be proportional (through $\mathcal{W}$ ) to the zero helicity component of $\mathbf{I}_{a b c d}$; indeed, by setting $(a, b, e, f)=(1,1,2,2)$ in $(3.12)$, one obtains:

$$
\frac{1}{2} \mathbf{I}_{0} \mathcal{W}=\frac{1}{4}\left\langle\widetilde{\mathcal{Q}}_{111}, \widetilde{\mathcal{Q}}_{222}\right\rangle-\frac{3}{4}\left\langle\widetilde{\mathcal{Q}}_{122}, \widetilde{\mathcal{Q}}_{112}\right\rangle
$$

Furthermore, at least in reducible symmetric models (listed in table 1), the sextic invariant is naturally defined within the $\mathbb{T}$-tensor formalism as follows (see section 3 of [44] for further detail):

$$
\mathbf{I}_{6}^{\prime}=-\operatorname{Tr}_{\eta}\left(\mathbb{T}_{11} \mathbb{T}_{22} \mathbb{T}_{12}\right)
$$

As denoted by the prime, $\mathbf{I}_{6}^{\prime}(3.17)$ does not coincide with the $\mathbf{I}_{6}$ given by (3.14) (or equivalently by (3.15)); indeed, irreducible and reducible symmetric models, the following relation respectively holds:

$$
\begin{aligned}
& \mathbf{I}_{6}=\mathbf{I}_{6}^{\prime}+\frac{1}{12} \mathcal{W}^{3} \\
& \mathbf{I}_{6}=\mathbf{I}_{6}^{\prime}+\frac{1}{12} \mathcal{W}^{3}+\frac{1}{6} \mathcal{X} \mathcal{W} .
\end{aligned}
$$

This can be traced back to a crucial difference (pointed out in section 3 of [45]) between reducible and irreducible symmetric models, concerning the horizontal invariant polynomial (of degree four in charges) $\mathcal{X}$. In reducible models, $\mathcal{X}$ is defined by eq. (4.13) of [44]:

$$
\mathcal{X} \equiv \operatorname{Tr}_{\eta}\left(\mathbb{T}_{12}^{2}\right)-\operatorname{Tr}_{\eta}\left(\mathbb{T}_{11} \mathbb{T}_{22}\right)-\frac{1}{2} \mathcal{W}^{2}
$$

and it generally does not vanish. On the other hand, in irreducible models it is defined by eq. (3.10) of [45], and it vanishes identically:

$$
\mathcal{X}_{\text {irred }}=0
$$

Thus, it is here worth remarking that the relations (3.11)-(3.16) hold both in irreducible models (characterized by (3.21) and in reducible models (generally having non-vanishing $\mathcal{X}(3.20))$. On the other hand, in presence of $\mathcal{X} \neq 0, \mathbf{I}_{6}^{\prime}$ undergoes the renormalization $\mathbf{I}_{6}^{\prime} \rightarrow \mathbf{I}_{6}^{\prime}+\frac{1}{6} \mathcal{X} \mathcal{W}$, and this explains eq. (3.19) from eq. (3.18).

Before analyzing and classifying the two-center extremal $\mathrm{BH}$ configurations and the corresponding defining constraints in terms of $G_{4^{-}}$and $\left[S L_{h}(2, \mathbb{R}) \times G_{4}\right]$ - invariant polynomials, in light of previous definitions and findings, we conclude this section by pointing out some consequences of the rank of a charge vector, say $\mathcal{Q}_{1}$, on the set of $G_{4^{-}}$and $\left[S L_{h}(2, \mathbb{R}) \times G_{4}\right]$ - invariant polynomials of the two-centered configuration $\left(\mathcal{Q}_{1}, \mathcal{Q}_{2}\right)$, both 
in irreducible and reducible symmetric models:

$$
\begin{aligned}
& \operatorname{rank}\left(\mathcal{Q}_{1}\right)=3 \Rightarrow \mathbf{I}_{+2}=0 ; \\
& \operatorname{rank}\left(\mathcal{Q}_{1}\right)=2 \Rightarrow \widetilde{\mathcal{Q}}_{111}=0 \Rightarrow\left\{\begin{array}{l}
\mathbf{I}_{+2}=\mathbf{I}_{+1}=0 \\
\mathbf{I}_{6}^{\prime}=-\frac{1}{2} \mathbf{I}_{0} \mathcal{W}-\frac{1}{12} \mathcal{W}^{3}-\frac{1}{6} \mathcal{X} \mathcal{W} \Leftrightarrow \mathbf{I}_{6}=-\frac{1}{2} \mathbf{I}_{0} \mathcal{W}
\end{array}\right. \\
& \operatorname{rank}\left(\mathcal{Q}_{1}\right)=1 \Rightarrow\left\{\begin{array}{l}
\mathbf{I}_{+2}=\mathbf{I}_{+1}=0 ; \\
\mathbf{I}_{0}=-\frac{1}{6} \mathcal{W}^{2} ; \\
\mathbf{I}_{6}^{\prime}=0 \Leftrightarrow \mathbf{I}_{6}=-\frac{1}{2} \mathbf{I}_{0} \mathcal{W}=\frac{1}{12} \mathcal{W}^{3} ; \\
\mathcal{X}=0 \text { (in reducible models). }
\end{array}\right.
\end{aligned}
$$

Ça va sans dire that analogous relations, involving components of opposite horizontal helicity, hold for $\mathcal{Q}_{2}$. Eqs. (3.22)-(3.24) will be used extensively in sections 4 and 5 (as given by (3.21), $\mathcal{X}=0$ identically in reducible models). The non-generic cases of $s t^{2}$ and $t^{3} \mathcal{N}=2, d=4$ models will be be considered in sections 4.2 and 5.2, respectively.

\section{Reducible models}

As given by table 1 , the reducible symmetric $d=4$ supergravity models ${ }^{5}$ have the following $d=4 U$-duality group:

$$
\begin{gathered}
G_{4}=S L_{v}(2, \mathbb{R}) \times S O(m, n) \\
\mathbb{i} \\
S L_{h}(2, \mathbb{R}) \times G_{4} \sim S O_{h}^{v}(2,2) \times S O(m, n),
\end{gathered} \quad m=\left\{\begin{array}{l}
2(\mathcal{N}=2, n \in \mathbb{N}) ; \\
6(\mathcal{N}=6, n \in \mathbb{N} \cup\{0\}),
\end{array}\right.
$$

where the isomorphism (see section 8 of [44])

$$
S L_{h}(2, \mathbb{R}) \times S L_{v}(2, \mathbb{R}) \sim S O_{h}^{v}(2,2)
$$

has been used. The corresponding scalar manifolds thus belong to the sequence $\mathcal{S T}[m, n]$, of particular relevance for superstring compactifications (see e.g. the analysis in section 3.1 and appendix $\mathrm{C}$ of [83], and refs. therein).

We now give a complete analysis of all possible two-center charge configurations $\left(\mathcal{Q}_{1}, \mathcal{Q}_{2}\right)$ (with symplectic product $(1.2) \mathcal{W} \neq 0$, i.e. mutually non-local), by providing for each configuration a "minimal" sets of independent $G_{4}$-invariant and $\left[S L_{h}(2, \mathbb{R}) \times G_{4}\right]$ invariant polynomials. The analysis will be carried out in the bare charges $\mathcal{Q}$ basis, by exploiting, for each of the two centers, the duality-invariant definitions of rank of $\mathcal{Q}$ recalled in section 2. The definitions and notation of [44] are used. The prototype of a generic $(\mathcal{N}=2)$ reducible symmetric model is the stu model, studied in some detail in section 2 of [44]. The non-generic case of the $\mathcal{N}=2, d=4 s t^{2}$ model is considered in section 4.2.

\footnotetext{
${ }^{5}$ Marginal stability for these models was studied e.g. in [12, 21].
} 


\subsection{Generic reducible models}

1. $\left(\mathcal{Q}_{1}, \mathcal{Q}_{2}\right)=(\operatorname{rank} 4, \operatorname{rank} 4)$. This is the configuration supporting the generic 2 center charge orbits, studied in some detail in [44]. The number of independent $G_{4}$-invariant is seven, a complete basis can be taken to be given by eq. (1.15). On the other hand, the number of independent $\left[S L_{h}(2, \mathbb{R}) \times G_{4}\right]$-invariant is four, and one can choose a complete basis to be [44]

$$
\left[S L_{h}(2, \mathbb{R}) \times G_{4}\right]-\operatorname{inv}(\#=4): \mathcal{W}, \mathcal{X}, \operatorname{Tr}\left(\mathfrak{I}^{2}\right), \operatorname{Tr}\left(\mathfrak{I}^{3}\right),
$$

where

$$
\begin{aligned}
& \operatorname{Tr}\left(\mathfrak{I}^{2}\right)=\mathbf{I}_{+2} \mathbf{I}_{-2}+3 \mathbf{I}_{0}^{2}-4 \mathbf{I}_{+1} \mathbf{I}_{-1} ; \\
& \operatorname{Tr}\left(\mathfrak{I}^{3}\right)=\mathbf{I}_{0}^{3}+\mathbf{I}_{+2} \mathbf{I}_{-1}^{2}+\mathbf{I}_{-2} \mathbf{I}_{+1}^{2}-\mathbf{I}_{+2} \mathbf{I}_{-2} \mathbf{I}_{0}-2 \mathbf{I}_{+1} \mathbf{I}_{-1} \mathbf{I}_{0} .
\end{aligned}
$$

Different choices are of course possible. E.g., equivalent duality-invariant and horizontal-invariant complete bases respectively read

$$
\begin{aligned}
& G_{4} \text {-inv }: \mathcal{W}, \mathcal{X}, \mathbf{I}_{6}^{\prime}, \mathbf{I}_{ \pm 2}, \mathcal{I}_{4}\left(\mathcal{Q}_{1}+\mathcal{Q}_{2}\right), \operatorname{Tr}\left(\mathfrak{I}^{2}\right) \\
& {\left[S L_{h}(2, \mathbb{R}) \times G_{4}\right] \text {-inv }: \mathcal{W}, \mathcal{X}, \mathbf{I}_{6}^{\prime}, \operatorname{Tr}\left(\mathfrak{I}^{2}\right) }
\end{aligned}
$$

The choice (4.6)-(4.7) is characterized by the fact that the horizontal basis is a subset of the duality basis. Furthermore, the duality basis contains quantities related both to the single-center BH entropy $\pi \sqrt{\left|\mathcal{I}_{4}\left(\mathcal{Q}_{1}+\mathcal{Q}_{2}\right)\right|}$ and to the two-centered BH entropy (1.4). In general, $\mathbf{I}_{6}^{\prime}$ is related to the elements of the basis (4.3) by means of the polynomial constraint of degree twelve in charges given by eq. (5.6) of [44], which we recall here (see also the analysis in [46]):

$$
\begin{aligned}
\left(\mathbf{I}_{6}^{\prime}\right)^{2}+\mathcal{W} \mathcal{X} \mathbf{I}_{6}^{\prime}+\operatorname{Tr}\left(\mathfrak{I}^{3}\right)+\frac{1}{12} \mathcal{W}^{2} \operatorname{Tr} & \left(\mathfrak{I}^{2}\right)-\frac{1}{3} \mathcal{X} \operatorname{Tr}\left(\mathfrak{I}^{2}\right)-\frac{1}{432} \mathcal{W}^{6} \\
+ & \frac{1}{36} \mathcal{X} \mathcal{W}^{4}+\frac{5}{36} \mathcal{W}^{2} \mathcal{X}^{2}+\frac{4}{27} \mathcal{X}^{3}=0 .
\end{aligned}
$$

2. $\left(\mathcal{Q}_{1}, \mathcal{Q}_{2}\right)=($ rank 3 , rank 4$)$. The complete duality and horizontal bases can respectively be taken to be

$$
\begin{aligned}
& G_{4} \text {-inv }(\#=6): \mathcal{W}, \mathcal{X}, \mathbf{I}_{-2}, \mathbf{I}_{ \pm 1}, \mathbf{I}_{0} \\
& {\left[S L_{h}(2, \mathbb{R}) \times G_{4}\right]-\operatorname{inv}(\#=4): \mathcal{W}, \mathcal{X}, \operatorname{Tr}\left(\mathfrak{I}^{2}\right), \operatorname{Tr}\left(\mathfrak{I}^{3}\right) }
\end{aligned}
$$

where now

$$
\begin{aligned}
& \operatorname{Tr}\left(\mathfrak{I}^{2}\right)=3 \mathbf{I}_{0}^{2}-4 \mathbf{I}_{+1} \mathbf{I}_{-1} ; \\
& \operatorname{Tr}\left(\mathfrak{I}^{3}\right)=\mathbf{I}_{0}^{3}+\mathbf{I}_{-2} \mathbf{I}_{+1}^{2}-2 \mathbf{I}_{+1} \mathbf{I}_{0} \mathbf{I}_{-1} .
\end{aligned}
$$

3. $\left(\mathcal{Q}_{1}, \mathcal{Q}_{2}\right)=(\operatorname{rank} 3, \operatorname{rank} 3)$ :

$$
\begin{aligned}
G_{4} \text {-inv }(\#=5) & : \mathcal{W}, \mathcal{X}, \mathbf{I}_{ \pm 1}, \mathbf{I}_{0} \\
{\left[S L_{h}(2, \mathbb{R}) \times G_{4}\right]-\operatorname{inv}(\#=4) } & : \mathcal{W}, \mathcal{X}, \operatorname{Tr}\left(\mathfrak{I}^{2}\right), \operatorname{Tr}\left(\mathfrak{I}^{3}\right),
\end{aligned}
$$


where $\operatorname{Tr}\left(\mathfrak{I}^{2}\right)$ is given by (4.11), and $\operatorname{Tr}\left(\mathfrak{I}^{3}\right)$ is further simplified to

$$
\operatorname{Tr}\left(\mathfrak{I}^{3}\right)=\mathbf{I}_{0}\left(\mathbf{I}_{0}^{2}-2 \mathbf{I}_{+1} \mathbf{I}_{-1}\right) .
$$

4. $\left(\mathcal{Q}_{1}, \mathcal{Q}_{2}\right)=(\operatorname{rank} 2, \operatorname{rank} 4)$ :

$$
\begin{aligned}
G_{4} \text {-inv }(\#=4) & : \mathcal{W}, \mathcal{X}, \mathbf{I}_{-2}, \mathbf{I}_{-1} \\
{\left[S L_{h}(2, \mathbb{R}) \times G_{4}\right]-\operatorname{inv}(\#=2) } & : \mathcal{W}, \mathcal{X} .
\end{aligned}
$$

This case actually splits in two subcases. Indeed, by plugging (3.23) into (4.8), this latter factorizes as

$$
\left(\mathcal{W}^{2}-4 \mathcal{X}+6 \mathbf{I}_{0}\right)\left(\mathcal{W}^{2}+2 \mathcal{X}+6 \mathbf{I}_{0}\right)=0
$$

thus admitting two solutions:

$$
\begin{aligned}
& I:\left\{\begin{array}{l}
\mathbf{I}_{0}=-\frac{1}{6} \mathcal{W}^{2}+\frac{2}{3} \mathcal{X} \\
\mathbf{I}_{6}^{\prime}=-\frac{1}{2} \mathcal{X} \mathcal{W} ; \\
\operatorname{Tr}\left(\mathfrak{I}^{2}\right)=3 \mathbf{I}_{0}^{2}=\frac{1}{3}\left(\frac{1}{4} \mathcal{W}^{4}+4 \mathcal{X}^{2}-2 \mathcal{W}^{2} \mathcal{X}\right) ; \\
\operatorname{Tr}\left(\mathfrak{I}^{3}\right)=\mathbf{I}_{0}^{3}=\frac{1}{3}\left(-\frac{1}{72} \mathcal{W}^{6}+\frac{8}{9} \mathcal{X}^{3}+\frac{1}{2} \mathcal{W}^{4} \mathcal{X}-\frac{2}{3} \mathcal{W}^{2} \mathcal{X}^{2}\right) .
\end{array}\right. \\
& I I:\left\{\begin{array}{l}
\mathbf{I}_{0}=-\frac{1}{6} \mathcal{W}^{2}-\frac{1}{3} \mathcal{X} \\
\mathbf{I}_{6}^{\prime}=0 ; \\
\operatorname{Tr}\left(\Im^{2}\right)=3 \mathbf{I}_{0}^{2}=\frac{1}{3}\left(\frac{1}{4} \mathcal{W}^{4}+\mathcal{X}^{2}+\mathcal{W}^{2} \mathcal{X}\right) ; \\
\operatorname{Tr}\left(\Im^{3}\right)=\mathbf{I}_{0}^{3}=-\frac{1}{9}\left(\frac{1}{24} \mathcal{W}^{6}+\frac{1}{3} \mathcal{X}^{3}+\frac{1}{4} \mathcal{W}^{4} \mathcal{X}+\frac{1}{2} \mathcal{W}^{2} \mathcal{X}^{2}\right) .
\end{array}\right.
\end{aligned}
$$

5. $\left(\mathcal{Q}_{1}, \mathcal{Q}_{2}\right)=(\operatorname{rank} 2, \operatorname{rank} 3)$ :

$$
\begin{aligned}
G_{4} \text {-inv }(\#=3) & : \mathcal{W}, \mathcal{X}, \mathbf{I}_{-1} \\
{\left[S L_{h}(2, \mathbb{R}) \times G_{4}\right]-\operatorname{inv}(\#=2) } & : \mathcal{W}, \mathcal{X} .
\end{aligned}
$$

The same splitting into subcases $I$ and $I I$, as given by (4.19) and (4.20), occurs.

6. $\left(\mathcal{Q}_{1}, \mathcal{Q}_{2}\right)=(\operatorname{rank} 2$, rank 2$)$. By recalling (3.23), the complete duality and horizontal bases can be taken to coincide:

$$
G_{4} \text {-inv and }\left[S L_{h}(2, \mathbb{R}) \times G_{4}\right] \operatorname{-inv}(\#=2): \mathcal{W}, \mathcal{X} .
$$

The same splitting into subcases $I$ and $I I$, as given by (4.19) and (4.20), occurs. For this configuration, a third subcase $I I I$ turns out to occur, namely:

$$
I I I:\left\{\begin{array}{l}
\mathcal{X}=0 ; \\
\mathbf{I}_{6}^{\prime}=0 ; \\
\mathbf{I}_{0}=-\frac{1}{6} \mathcal{W}^{2}<0 ; \\
\operatorname{Tr}\left(\mathfrak{I}^{2}\right)=3 \mathbf{I}_{0}^{2}=\frac{1}{12} \mathcal{W}^{4} ; \\
\operatorname{Tr}\left(\mathfrak{I}^{3}\right)=\mathbf{I}_{0}^{3}=-\frac{1}{216} \mathcal{W}^{6} .
\end{array}\right.
$$

Thus, for subcase $I I I(4.24) \mathcal{W}$ is the only relevant polynomial invariant. 
7. $\left(\mathcal{Q}_{1}, \mathcal{Q}_{2}\right)=(\operatorname{rank} 1, \operatorname{rank} 4)$ :

$$
\begin{aligned}
G_{4}-\operatorname{inv}(\#=3) & : \mathcal{W}, \mathbf{I}_{-2}, \mathbf{I}_{-1} \\
{\left[S L_{h}(2, \mathbb{R}) \times G_{4}\right]-\operatorname{inv}(\#=1): } & \mathcal{W} .
\end{aligned}
$$

Indeed, for this configuration (3.24) trivially implies (4.24). Thus, the configurations $\left(\mathcal{Q}_{1}, \mathcal{Q}_{2}\right)=(\operatorname{rank} 2, \operatorname{rank} 2)($ subcase $I I I)$ and $\left(\mathcal{Q}_{1}, \mathcal{Q}_{2}\right)=(\operatorname{rank} 1$, rank 4$)$ both implies (4.24), but they do differ at the level of $G_{4}$-invariant polynomials: in the former case only $\mathcal{W}$ matters, whereas in the latter case, as given by (4.25), the complete duality basis is three-dimensional.

8. $\left(\mathcal{Q}_{1}, \mathcal{Q}_{2}\right)=(\operatorname{rank} 1, \operatorname{rank} 3)$ :

$$
\begin{aligned}
G_{4} \text {-inv }(\#=2) & : \mathcal{W}, \mathbf{I}_{-1} \\
{\left[S L_{h}(2, \mathbb{R}) \times G_{4}\right]-\operatorname{inv}(\#=1) } & : \mathcal{W},
\end{aligned}
$$

with eq. (4.24) holding true.

9. $\left(\mathcal{Q}_{1}, \mathcal{Q}_{2}\right)=(\operatorname{rank} 1$, rank 2). By recalling (3.23) and (3.24), one obtains that the only relevant polynomial invariant is the symplectic product $\mathcal{W}$ :

$$
G_{4} \text {-inv and }\left[S L_{h}(2, \mathbb{R}) \times G_{4}\right]-\operatorname{inv}(\#=1): \mathcal{W},
$$

with eq. (4.24) holding true.

10. $\left(\mathcal{Q}_{1}, \mathcal{Q}_{2}\right)=($ rank $1, \operatorname{rank} 1)$. eq. (4.24) still holds true, and one obtains that the only relevant polynomial invariant is the symplectic product $\mathcal{W}$. An example of this configuration is the $D 0+D 6$ two-constituents solution of the stu model studied in [11] (see also [9]).

\subsection{The $s t^{2}$ model}

We now proceed to consider the non-generic case of the $\mathcal{N}=2, d=4 s t^{2}$ model, which has a rank-2 vector multiplets' scalar manifold, namely $\left[\frac{S L(2, \mathbb{R})}{U(1)}\right]^{2}$, first $(n=1)$ element of the $\mathcal{N}=2$ sequence in table 1 . Thus, the $U$-duality group is

$$
G_{4, s t^{2}}=S L_{v}(2, \mathbb{R}) \times S O(2,1) \sim S L_{v}(2, \mathbb{R}) \times S L(2, \mathbb{R}) .
$$

This model may be obtained as a rank-2 truncation (also named "st ${ }^{2}$ degeneration"; see e.g. $[44,66,67])$ of the prototype of generic reducible $\mathcal{N}=2$ symmetric models which, as observed above, is the stu model.

1. $\left(\mathcal{Q}_{1}, \mathcal{Q}_{2}\right)=($ rank 4 , rank 4$)$. This is the configuration supporting the generic 2 center orbit, studied (in the BPS case) in some detail in section 6 of [44]. The number of independent $G_{4}$-invariant is six, a complete basis can be taken to be [44]

$$
\left[S L_{v}(2, \mathbb{R}) \times S O(2,1)\right] \operatorname{-inv}(\#=6): \mathcal{W}, \mathcal{X}, \mathbf{I}_{ \pm 2}, \mathbf{I}_{ \pm 1}
$$


On the other hand, the number of independent $\left[S L_{h}(2, \mathbb{R}) \times G_{4}\right]$-invariant is three, and a complete basis can be taken to be [44]

$$
\left[S O_{h}^{v}(2,2) \times S O(2,1)\right]-\operatorname{inv}(\#=3): \mathcal{W}, \mathcal{X}, \operatorname{Tr}\left(\mathfrak{I}^{2}\right)
$$

Two polynomial constraints, of degree sixteen and eight in charges, hold in the $s t^{2}$ model; they are given by eqs. (6.10)-(6.12) of [44], which we recall here (see also the analysis in [46]):

$$
\begin{aligned}
0= & 16 \operatorname{Tr}^{2}\left(\mathfrak{I}^{2}\right)+64 \operatorname{Tr}\left(\mathfrak{I}^{3}\right) \mathcal{W}^{2}+\frac{8}{3} \operatorname{Tr}\left(\mathfrak{I}^{2}\right) \mathcal{W}^{4}-\frac{1}{27} \mathcal{W}^{8} \\
& -\frac{32}{3} \operatorname{Tr}\left(\mathfrak{I}^{2}\right) \mathcal{X}^{2}+\frac{8}{9} \mathcal{W}^{4} \mathcal{X}^{2}+\frac{64}{27} \mathcal{W}^{2} \mathcal{X}^{3}+\frac{16}{9} \mathcal{X}^{4} \\
0= & 12 \operatorname{Tr}\left(\mathfrak{I}^{2}\right)-24 \mathbf{I}_{6}^{\prime} \mathcal{W}-\left(\mathcal{W}^{2}+2 \mathcal{X}\right)^{2} .
\end{aligned}
$$

By means of (4.33) and (4.34), $\mathbf{I}_{6}^{\prime}$ and $\operatorname{Tr}\left(\mathfrak{I}^{3}\right)$ can be expressed in terms of the horizontal invariants of the basis (4.32).

2. $\left(\mathcal{Q}_{1}, \mathcal{Q}_{2}\right)=(\operatorname{rank} 3, \operatorname{rank} 4)$ :

$$
\begin{gathered}
{\left[S L_{v}(2, \mathbb{R}) \times S O(2,1)\right]-\operatorname{inv}(\#=5): \mathcal{W}, \mathcal{X}, \mathbf{I}_{-2}, \mathbf{I}_{ \pm 1}} \\
{\left[S O_{h}^{v}(2,2) \times S O(2,1)\right]-\operatorname{inv}(\#=3): \mathcal{W}, \mathcal{X}, \operatorname{Tr}\left(\mathfrak{I}^{2}\right),}
\end{gathered}
$$

with (4.11) and (4.12) holding true, as well.

3. $\left(\mathcal{Q}_{1}, \mathcal{Q}_{2}\right)=(\operatorname{rank} 3$, rank 3$)$ :

$$
\begin{aligned}
& {\left[S L_{v}(2, \mathbb{R}) \times S O(2,1)\right]-\operatorname{inv}(\#=4): \mathcal{W}, \mathcal{X}, \mathbf{I}_{ \pm 1}} \\
& {\left[S O_{h}^{v}(2,2) \times S O(2,1)\right]-\operatorname{inv}(\#=3): \mathcal{W}, \mathcal{X}, \operatorname{Tr}\left(\mathfrak{I}^{2}\right)}
\end{aligned}
$$

where $\operatorname{Tr}\left(\mathfrak{I}^{2}\right)$ is given by $(4.11)$ and $\operatorname{Tr}\left(\mathfrak{I}^{3}\right)$ is further simplified to (4.15).

4. $\left(\mathcal{Q}_{1}, \mathcal{Q}_{2}\right)=(\operatorname{rank} 2, \operatorname{rank} 4)$ :

$$
\begin{aligned}
& {\left[S L_{v}(2, \mathbb{R}) \times S O(2,1)\right]-\operatorname{inv}(\#=4): \mathcal{W}, \mathcal{X}, \mathbf{I}_{-2}, \mathbf{I}_{-1}} \\
& {\left[S O_{h}^{v}(2,2) \times S O(2,1)\right]-\operatorname{inv}(\#=2): \mathcal{W}, \mathcal{X}}
\end{aligned}
$$

The splitting into subcases $I$ (4.19) and $I I$ (4.20), characterizing the generic reducible models (see e.g. point 4 of section 4 , does not occur in the $s t^{2}$ model. Indeed, in such a model the unique solution of the polynomial constraints (4.33) and (4.34) for $\left(\mathcal{Q}_{1}, \mathcal{Q}_{2}\right)=($ rank 2 , rank 4$)$ is given by eq. (4.20) (namely, only case $I I$ holds).

5. $\left(\mathcal{Q}_{1}, \mathcal{Q}_{2}\right)=(\operatorname{rank} 2, \operatorname{rank} 3)$ :

$$
\begin{aligned}
& {\left[S L_{v}(2, \mathbb{R}) \times S O(2,1)\right]-\operatorname{inv}(\#=3): \mathcal{W}, \mathcal{X}, \mathbf{I}_{-1}} \\
& {\left[S O_{h}^{v}(2,2) \times S O(2,1)\right]-\operatorname{inv}(\#=2): \mathcal{W}, \mathcal{X} .}
\end{aligned}
$$


6. $\left(\mathcal{Q}_{1}, \mathcal{Q}_{2}\right)=(\operatorname{rank} 2, \operatorname{rank} 2)$ :

$$
\left[S L_{v}(2, \mathbb{R}) \times S O(2,1)\right]-\operatorname{inv} \text { and }\left[S O_{h}^{v}(2,2) \times S O(2,1)\right]-\operatorname{inv}(\#=2): \mathcal{W}, \mathcal{X} .
$$

For this configuration, there is actually a second subcase given by the subcase III (4.24) of the generic models, in which then $\mathcal{W}$ is the only relevant polynomial invariant. In the $s t^{2}$ model, $(4.24)$ is realized in the configuration $\left(\mathcal{Q}_{1}, \mathcal{Q}_{2}\right)=$ (rank 2, rank 2) for instance by setting the charges of the two vector multiplets to coincide; in the " $d=4 / d=5$ special coordinates" symplectic frame, a particular realization of this is the " $t^{3}$ degeneration" (see e.g. $[44,66,67]$ ) in which $p^{1}=p^{2} \equiv p$ and $q_{1}=q_{2} \equiv q / 2$.

7. $\left(\mathcal{Q}_{1}, \mathcal{Q}_{2}\right)=(\operatorname{rank} 1, \operatorname{rank} 4)$ :

$$
\begin{aligned}
& {\left[S L_{v}(2, \mathbb{R}) \times S O(2,1)\right]-\operatorname{inv}(\#=3): \mathcal{W}, \mathbf{I}_{-2}, \mathbf{I}_{-1}} \\
& {\left[S O_{h}^{v}(2,2) \times S O(2,1)\right]-\operatorname{inv}(\#=1): \mathcal{W} .}
\end{aligned}
$$

Indeed, also in the $s t^{2}$ model eq. (4.24) holds true.

8. $\left(\mathcal{Q}_{1}, \mathcal{Q}_{2}\right)=(\operatorname{rank} 1, \operatorname{rank} 3)$. As for the generic reducible models, eq. (4.24) does hold for $\mathcal{Q}_{1}$, and the complete duality and horizontal bases can respectively be taken to be

$$
\begin{aligned}
& {\left[S L_{v}(2, \mathbb{R}) \times S O(2,1)\right]-\operatorname{inv}(\#=2): \mathcal{W}, \mathbf{I}_{-1}} \\
& {\left[S O_{h}^{v}(2,2) \times S O(2,1)\right]-\operatorname{inv}(\#=1): \mathcal{W} .}
\end{aligned}
$$

9. $\left(\mathcal{Q}_{1}, \mathcal{Q}_{2}\right)=($ rank 1 , rank 2$)$. eq. (4.24) does hold for $\mathcal{Q}_{1}$, and the only relevant polynomial invariant is $\mathcal{W}$, as given by eq. (4.29).

10. $\left(\mathcal{Q}_{1}, \mathcal{Q}_{2}\right)=$ (rank 1, rank 1$)$. eq. (4.24) does hold for $\mathcal{Q}_{1}$ and for $\mathcal{Q}_{2}$, and eq. (4.29) also holds, i.e. the only relevant polynomial invariant is $\mathcal{W}$.

\section{$5 \quad$ Irreducible models}

We now proceed to consider the irreducible symmetric models (see table 2), by providing a complete analysis of all possible two-center charge configurations $\left(\mathcal{Q}_{1}, \mathcal{Q}_{2}\right)$ (with symplectic product $(1.2) \mathcal{W} \neq 0$, i.e. mutually non-local), in the very same way as done for reducible symmetric models in section 4 . The definitions and notation of [44] and [45] are used. The non-generic case of the $\mathcal{N}=2, d=4 t^{3}$ model is considered in section 5.2.

\subsection{Generic irreducible models}

1. $\left(\mathcal{Q}_{1}, \mathcal{Q}_{2}\right)=(\operatorname{rank} 4, \operatorname{rank} 4)$. This is the configuration supporting the generic 2 center orbits, studied in some detail in [45]. As mentioned in section 3 and derived in [45] (see eq. (3.10) therein), the quartic horizontal-invariant polynomial $\mathcal{X}_{\text {irred }}$ vanishes identically in the class of models under consideration. Furthermore, (an 
analogue with $\mathcal{X}_{\text {irred }}=0$ of) the polynomial constraint (4.8) does not hold in these models. As discussed in [45], the number of independent $G_{4}$-invariant is seven, a complete basis can be taken to be given by eq. (1.16). On the other hand, the number of independent $\left[S L_{h}(2, \mathbb{R}) \times G_{4}\right]$-invariant is four, and one can choose the following complete basis [45]:

$$
\left[S L_{h}(2, \mathbb{R}) \times G_{4}\right]-\operatorname{inv}(\#=4): \mathcal{W}, \mathbf{I}_{6}, \operatorname{Tr}\left(\mathfrak{I}^{2}\right), \operatorname{Tr}\left(\mathfrak{I}^{3}\right) .
$$

As for the reducible models analyzed in section 4, different choices are of course possible. E.g., an equivalent duality-invariant complete basis respectively reads

$$
G_{4} \text {-inv }: \mathcal{W}, \mathbf{I}_{6}, \mathbf{I}_{ \pm 2}, \mathcal{I}_{4}\left(\mathcal{Q}_{1}+\mathcal{Q}_{2}\right), \operatorname{Tr}\left(\mathfrak{I}^{2}\right), \operatorname{Tr}\left(\mathfrak{I}^{3}\right) .
$$

The choice (5.2) is characterized by the fact that the horizontal basis is a subset of the duality basis. Furthermore, in this case the duality basis contains quantities related both to the single-center $\mathrm{BH}$ entropy and to the two-centered $\mathrm{BH}$ entropy.

2. $\left(\mathcal{Q}_{1}, \mathcal{Q}_{2}\right)=(\operatorname{rank} 3, \operatorname{rank} 4)$ :

$$
\begin{aligned}
G_{4} \text {-inv }(\#=6): \mathcal{W}, \mathbf{I}_{6}, \mathbf{I}_{-2}, \mathbf{I}_{ \pm 1}, \mathbf{I}_{0} \\
{\left[S L_{h}(2, \mathbb{R}) \times G_{4}\right]-\operatorname{inv}(\#=4): \mathcal{W}, \mathbf{I}_{6}, \operatorname{Tr}\left(\mathfrak{I}^{2}\right), \operatorname{Tr}\left(\mathfrak{I}^{3}\right), }
\end{aligned}
$$

where $\operatorname{Tr}\left(\mathfrak{I}^{2}\right)$ and $\operatorname{Tr}\left(\mathfrak{I}^{3}\right)$ are respectively given by (4.11) and (4.12).

3. $\left(\mathcal{Q}_{1}, \mathcal{Q}_{2}\right)=(\operatorname{rank} 3$, rank 3$)$ :

$$
\begin{aligned}
G_{4} \text {-inv }(\#=5): \mathcal{W}, \mathbf{I}_{6}, \mathbf{I}_{ \pm 1}, \mathbf{I}_{0} ; \\
{\left[S L_{h}(2, \mathbb{R}) \times G_{4}\right]-\operatorname{inv}(\#=4): \mathcal{W}, \mathbf{I}_{6}, \operatorname{Tr}\left(\mathfrak{I}^{2}\right), \operatorname{Tr}\left(\mathfrak{I}^{3}\right), }
\end{aligned}
$$

where $\operatorname{Tr}\left(\mathfrak{I}^{2}\right)$ and $\operatorname{Tr}\left(\mathfrak{I}^{3}\right)$ are respectively given by (4.11) and (4.15).

4. $\left(\mathcal{Q}_{1}, \mathcal{Q}_{2}\right)=(\operatorname{rank} 2, \operatorname{rank} 4)$ :

$$
\begin{aligned}
G_{4} \text {-inv }(\#=4): \mathcal{W}, \mathbf{I}_{-2}, \mathbf{I}_{-1}, \mathbf{I}_{0} \\
{\left[S L_{h}(2, \mathbb{R}) \times G_{4}\right]-\operatorname{inv}(\#=2): \mathcal{W}, \operatorname{Tr}\left(\mathfrak{I}^{2}\right)=3 \mathbf{I}_{0}^{2} . }
\end{aligned}
$$

Furthermore, the limit $\mathbf{I}_{+1}=0$ of (4.15) yields

$$
\operatorname{Tr}\left(\mathfrak{I}^{3}\right)=\mathbf{I}_{0}^{3} .
$$

Note that in generic irreducible generic models, differently from what occurs in generic reducible generic models (see e.g. point 4 of section 4.1 ), the splitting in subcases does not occur.

5. $\left(\mathcal{Q}_{1}, \mathcal{Q}_{2}\right)=(\operatorname{rank} 2$, rank 3$)$ :

$$
\begin{aligned}
G_{4} \text {-inv }(\#=3) & : \mathcal{W}, \mathbf{I}_{-1}, \mathbf{I}_{0} \\
{\left[S L_{h}(2, \mathbb{R}) \times G_{4}\right]-\operatorname{inv}(\#=2) } & : \mathcal{W}, \operatorname{Tr}\left(\mathfrak{I}^{2}\right)=3 \mathbf{I}_{0}^{2},
\end{aligned}
$$

with eq. (5.9) holding true. 
6. $\left(\mathcal{Q}_{1}, \mathcal{Q}_{2}\right)=(\operatorname{rank} 2, \operatorname{rank} 2)$ :

$$
\begin{aligned}
G_{4} \text {-inv }(\#=2) & : \mathcal{W}, \mathbf{I}_{0} \\
{\left[S L_{h}(2, \mathbb{R}) \times G_{4}\right]-\operatorname{inv}(\#=2) } & : \mathcal{W}, \operatorname{Tr}\left(\mathfrak{I}^{2}\right)=3 \mathbf{I}_{0}^{2},
\end{aligned}
$$

with eq. (5.9) holding true. Equivalently, as done for the same configuration in generic reducible models as well as in the $s t^{2}$ model (see point 6 of sections 4.1 and 4.2 , respectively), the complete duality and horizontal bases can be taken to coincide:

$$
G_{4} \text {-inv and }\left[S L_{h}(2, \mathbb{R}) \times G_{4}\right] \text {-inv }(\#=2): \mathcal{W}, \operatorname{Tr}\left(\mathfrak{I}^{2}\right)=3 \mathbf{I}_{0}^{2} .
$$

7. $\left(\mathcal{Q}_{1}, \mathcal{Q}_{2}\right)=($ rank $1, \operatorname{rank} 4)$. As for generic reducible models as well as for the $s t^{2}$ model (see point 7 of sections 4.1 and 4.2, respectively), by recalling (3.24), the complete duality and horizontal bases can respectively be taken to be

$$
\begin{aligned}
G_{4} \text {-inv }(\#=3) & : \mathcal{W}, \mathbf{I}_{-2}, \mathbf{I}_{-1} \\
{\left[S L_{h}(2, \mathbb{R}) \times G_{4}\right]-\operatorname{inv}(\#=1) } & : \mathcal{W},
\end{aligned}
$$

with $\mathbf{I}_{0}=-\frac{1}{6} \mathcal{W}^{2}<0$, and

$$
\begin{aligned}
& \operatorname{Tr}\left(\mathfrak{I}^{2}\right)=3 \mathbf{I}_{0}^{2}=\frac{1}{12} \mathcal{W}^{4} ; \\
& \operatorname{Tr}\left(\mathfrak{I}^{3}\right)=\mathbf{I}_{0}^{3}=-\frac{1}{216} \mathcal{W}^{6} .
\end{aligned}
$$

8. $\left(\mathcal{Q}_{1}, \mathcal{Q}_{2}\right)=(\operatorname{rank} 1, \operatorname{rank} 3)$. Eqs. (5.17)-(5.18) hold true, and, as for generic reducible models as well as for the $s t^{2}$ model (see point 8 of sections 4.1 and 4.2 , respectively), the complete duality and horizontal bases can respectively be taken to be

$$
\begin{aligned}
G_{4}-\operatorname{inv}(\#=2) & : \mathcal{W}, \mathbf{I}_{-1} \\
{\left[S L_{h}(2, \mathbb{R}) \times G_{4}\right]-\operatorname{inv}(\#=1) } & : \mathcal{W} .
\end{aligned}
$$

9. $\left(\mathcal{Q}_{1}, \mathcal{Q}_{2}\right)=(\operatorname{rank} 1$, rank 2). Eqs. (5.17)-(5.18) hold true, and, as for generic reducible models as well as for the $s t^{2}$ model (see point 9 of sections 4.1 and 4.2 , respectively), the only relevant polynomial invariant is $\mathcal{W}$, as given by eq. (4.29).

10. $\left(\mathcal{Q}_{1}, \mathcal{Q}_{2}\right)=($ rank 1, rank 1). Eqs. (5.17)-(5.18) hold true, and, as for generic reducible models as well as for the $s t^{2}$ model (see point 10 of sections 4.1 and 4.2, respectively), the only relevant polynomial invariant is $\mathcal{W}$, as given by eq. (4.29).

\subsection{The $t^{3}$ model}

We now proceed to consider the non-generic case of the $\mathcal{N}=2, d=4 t^{3}$ model, which has a rank-1 vector multiplets' scalar manifold, namely $\frac{S L(2, \mathbb{R})}{U(1)}$ (see the first line of table 2. Thus, the $U$-duality group is

$$
G_{4}=S L_{v}(2, \mathbb{R})
$$


As mentioned above, this model provides a simple yet interesting example, because it may be obtained both as the rank- 1 truncation of the reducible $\mathcal{N}=2$ symmetric models, as well as the first $(q=0)$, non-generic element of the sequence of irreducible $\mathcal{N}=2$ symmetric models, which contains the four rank-3 magical supergravity theories [76, 77].

1. $\left(\mathcal{Q}_{1}, \mathcal{Q}_{2}\right)=(\operatorname{rank} 4, \operatorname{rank} 4)$. This is the configuration supporting the generic 2 center orbit, ${ }^{6}$ studied (in the BPS case) in some detail in section 7 of [44] (see also the comment in the Introduction of [45]). The number of independent $G_{4}$-invariant is five, a complete basis can be taken to be [44]

$$
S L_{v}(2, \mathbb{R})-\operatorname{inv}(\#=5): \mathcal{W}, \mathbf{I}_{6}, \mathbf{I}_{ \pm 2}, \mathbf{I}_{+1} .
$$

However, other equivalent choices read

$$
S L_{v}(2, \mathbb{R})-\operatorname{inv}(\#=5):\left\{\begin{array}{l}
\mathcal{W}, \mathbf{I}_{6}, \mathbf{I}_{ \pm 2}, \mathbf{I}_{0} \\
\text { or } \\
\mathcal{W}, \mathbf{I}_{6}, \mathbf{I}_{ \pm 2}, \mathcal{I}_{4}\left(\mathcal{Q}_{1}+\mathcal{Q}_{2}\right)
\end{array}\right.
$$

On the other hand, the number of independent $\left[S L_{h}(2, \mathbb{R}) \times G_{4}\right]$-invariant is two, and a complete basis can be taken to be [44] (recall isomorphism (4.2))

$$
S O_{h}^{v}(2,2)-\operatorname{inv}(\#=2): \mathcal{W}, \mathbf{I}_{6} .
$$

Three polynomial constraints, of degree sixteen, eight and four in charges, hold in the $t^{3}$ model; they are given by eqs. (6.12), (7.18), (7.17) and (7.16) of [44] (see also appendix A therein, and the analysis in [46]), which we recall here:

$$
\begin{aligned}
0 & =16 \operatorname{Tr}^{2}\left(\mathfrak{I}^{2}\right)+64 \operatorname{Tr}\left(\mathfrak{I}^{3}\right) \mathcal{W}^{2}+\frac{8}{3} \operatorname{Tr}\left(\mathfrak{I}^{2}\right) \mathcal{W}^{4}-\frac{1}{27} \mathcal{W}^{8} ; \\
0 & =24 \mathbf{I}_{6}^{\prime} \mathcal{W}-12 \operatorname{Tr}\left(\mathfrak{I}^{2}\right)+\mathcal{W}^{4} ; \\
\mathcal{X} & =0 .
\end{aligned}
$$

Note that in $(5.25) \mathcal{X} \equiv \mathcal{X}_{\text {irred }}$, and its identical vanishing consistently characterizes the $t^{3}$ model as a non-generic irreducible symmetric model. Due to (5.25), $\mathbf{I}_{6}^{\prime}$ and $\mathbf{I}_{6}$ are related through eq. (3.18). By means of (5.25), (5.25) and (3.18), $\operatorname{Tr}\left(\mathfrak{I}^{2}\right)$ and $\operatorname{Tr}\left(\mathfrak{I}^{3}\right)$ can be expressed in terms of the horizontal invariants of the basis (5.24).

2. $\left(\mathcal{Q}_{1}, \mathcal{Q}_{2}\right)=(\operatorname{rank} 3, \operatorname{rank} 4)$ :

$$
\begin{aligned}
& S L_{v}(2, \mathbb{R})-\operatorname{inv}(\#=4): \mathcal{W}, \mathbf{I}_{6}, \mathbf{I}_{-2}, \mathbf{I}_{+1} ; \\
& S O_{h}^{v}(2,2)-\operatorname{inv}(\#=2): \mathcal{W}, \mathbf{I}_{6} .
\end{aligned}
$$

3. $\left(\mathcal{Q}_{1}, \mathcal{Q}_{2}\right)=(\operatorname{rank} 3, \operatorname{rank} 3)$ :

$$
\begin{aligned}
& S L_{v}(2, \mathbb{R})-\operatorname{inv}(\#=3): \mathcal{W}, \mathbf{I}_{6}, \mathbf{I}_{+1} ; \\
& S O_{h}^{v}(2,2)-\operatorname{inv}(\#=2): \mathcal{W}, \mathbf{I}_{6} .
\end{aligned}
$$

${ }^{6}$ This charge configuration has been considered in literature $[4,6]$ but, within $S L_{v}(2, \mathbb{R})$-invariant polynomials (see e.g. the second possible basis of (5.23)), the role of $\mathbf{I}_{6}$ is not completely clear yet (concerning this, see the recent study in [46]). 
4. $\left(\mathcal{Q}_{1}, \mathcal{Q}_{2}\right)=($ rank 2 , rank 4$)$. This charge configuration has been considered in [3]. The complete duality and horizontal bases can respectively be taken to be

$$
\begin{aligned}
& S L_{v}(2, \mathbb{R})-\operatorname{inv}(\#=2): \mathcal{W}, \mathbf{I}_{-2} ; \\
& S O_{h}^{v}(2,2)-\operatorname{inv}(\#=1): \mathcal{W} .
\end{aligned}
$$

By recalling (5.25)-(5.25), in the $t^{3}$ model the configuration $\left(\mathcal{Q}_{1}, \mathcal{Q}_{2}\right)=$ ( rank 2, rank 4) implies

$$
\left\{\begin{array}{l}
\mathbf{I}_{6}^{\prime}=0 \stackrel{(3.18)}{\Leftrightarrow} \mathbf{I}_{6}=\frac{1}{12} \mathcal{W}^{3} \\
\mathbf{I}_{0}=-\frac{1}{6} \mathcal{W}^{2}<0 ; \\
\operatorname{Tr}\left(\mathfrak{I}^{2}\right)=\frac{1}{12} \mathcal{W}^{4} \\
\operatorname{Tr}\left(\mathfrak{I}^{3}\right)=-\frac{1}{216} \mathcal{W}^{6} .
\end{array}\right.
$$

Consistent with the fact that the $t^{3}$ model can be obtained as a "rank-1 degeneration" (see e.g. [44, 66, 67]) of the reducible symmetric $\mathcal{N}=2, d=4$ models, (5.32) matches the $\mathcal{X}=0$ limit of subcases $I$ (4.19) and $I I$ (4.20) or, equivalently, it matches the subcase $I I I(4.24)$.

5. $\left(\mathcal{Q}_{1}, \mathcal{Q}_{2}\right)=(\operatorname{rank} 2$, rank 3$)$. The complete duality and horizontal bases can be taken to coincide, and $\mathcal{W}$ is the only relevant invariant polynomial in charges:

$$
S L_{v}(2, \mathbb{R}) \text {-inv and } S O_{h}^{v}(2,2) \text {-inv }(\#=1): \mathcal{W} \text {. }
$$

6. $\left(\mathcal{Q}_{1}, \mathcal{Q}_{2}\right)=($ rank 2, rank 2). Again, the complete duality and horizontal bases can be taken to coincide and be given by (5.33).

Since the $t^{3}$ model lacks of an independent doubly-critical "small" charge orbit (namely, criticality implies doubly-criticality in this model; see e.g. [84] for a recent account within a $d=3$ timelike-reduced formalism), the cases given by points $7,8,9$ and 10 are all missing for this model.

\section{A comment on bound states with negative discriminant}

The treatment given in sections 4 and 5 allows one to discuss in fair generality two-centered extremal BH compound states with a given value of $\mathcal{I}_{4}\left(\mathcal{Q}_{1}+\mathcal{Q}_{2}\right)$. Here, we would like to comment shortly on some two-centered charge configurations corresponding to a negative $\mathcal{I}_{4}\left(\mathcal{Q}_{1}+\mathcal{Q}_{2}\right)$, i.e. to a $\mathrm{BH}$ compound that, regarded as a single-centered solution, is a "large" non-BPS (extremal) BH.

By recalling the sum rule (cf. eq. (4.7) of [44])

$$
\mathcal{I}_{4}\left(\mathcal{Q}_{1}+\mathcal{Q}_{2}\right)=\mathbf{I}_{+2}+4 \mathbf{I}_{+1}+6 \mathbf{I}_{0}+4 \mathbf{I}_{-1}+\mathbf{I}_{-2},
$$

from the analysis of previous sections one can single out some two-charge configurations which necessarily imply

$$
\mathcal{I}_{4}\left(\mathcal{Q}_{1}+\mathcal{Q}_{2}\right)=-\mathcal{W}^{2}<0
$$


Note that in this case $\mathcal{I}_{4}\left(\mathcal{Q}_{1}+\mathcal{Q}_{2}\right)$ becomes horizontal invariant, as well.

For all symmetric reducible models (see table 1), these configurations are the ones with

$$
\text { reducible }: \operatorname{rank}\left(\mathcal{Q}_{1}, \mathcal{Q}_{2}\right)=\left\{\begin{array}{l}
(2,2)_{I I I} \\
(1,2) ; \\
(1,1)
\end{array}\right.
$$

namely the subcase $I I I$ of point 6 , and points 9 and 10 of section 4.1 (and analogue cases for the non-generic $s t^{2}$ model treated in section 4.2).

For generic irreducible models, these configurations are the ones in which at least one center is doubly-critical, namely the ones with

$$
\text { irreducible: } \operatorname{rank}\left(\mathcal{Q}_{1}, \mathcal{Q}_{2}\right)=\left\{\begin{array}{l}
(1,2) ; \\
(1,1),
\end{array}\right.
$$

i.e. points 9 and 10 of section 5.1. In the non-generic irreducible $t^{3}$ model, treated in the section 5.2, the unique configuration of the kind under consideration is given by $\operatorname{rank}\left(\mathcal{Q}_{1}, \mathcal{Q}_{2}\right)=(2,2)$ (point 6 above), because, as mentioned in section 5.2, in such a model criticality implies doubly-criticality.

As an illustrative example (which can be realized in heterotic string theory), let us consider the "small + small" two-centered charge configuration (in Calabi-Vesentini symplectic frame $[50] ; \Lambda=1, \ldots ., m+n)$

$$
\mathcal{Q}_{1} \equiv\left(p^{\Lambda}, 0\right) ; \quad \mathcal{Q}_{2} \equiv\left(0, Q_{\Lambda}\right)
$$

in $d=4$ supergravity coupled to $n$ vector multiplets $(\mathcal{N}=2$ and $\mathcal{N}=4$ theories are obtained for $m=2$ and 6 , respectively), implying that ${ }^{7}[51-54]$

$$
\mathcal{I}_{4}\left(\mathcal{Q}_{1}+\mathcal{Q}_{2}\right)=\mathcal{I}_{4}(p, Q)=p^{2} Q^{2}-(p \cdot Q)^{2} .
$$

From the analysis of single-centered charge orbits [42, 56, 85], the charge vector $\mathcal{Q}_{1}$ of $(6.5)$ enjoys the following properties, depending on the nature of the $S O(m, n)$-vector $p^{\Lambda}$ :

$$
\begin{aligned}
& p^{2} \equiv p^{\Lambda} p^{\Sigma} \eta_{\Lambda \Sigma}>0 \Rightarrow \mathcal{Q}_{1} \text { rank }=2, \frac{1}{2} \text {-BPS } \\
& p^{2}<0 \Rightarrow \mathcal{Q}_{1} \text { rank }=2, \text { non-BPS } \\
& p^{2}=0 \Rightarrow \mathcal{Q}_{1} \text { rank }=1, \frac{1}{2} \text {-BPS }
\end{aligned}
$$

and the same holds for $\mathcal{Q}_{2}$ of (6.5), by replacing $p^{\Lambda}$ with $Q_{\Lambda}$ ( $\eta$ is the $S O(m, n)$ metric). By using e.g. the formulæ derived of [44], one can easily compute that in the heterotic charge configuration (6.5) there unique two independent horizontal-invariant polynomials read:

$$
\mathcal{W}=-p \cdot Q \neq 0 ; \quad \mathcal{X}=-\frac{1}{2} p^{2} Q^{2} \lesseqgtr 0,
$$

where the two centers are assumed to have mutually non-local fluxes (and thus it is assumed that $\mathcal{W} \neq 0$ ).

\footnotetext{
${ }^{7}$ In string theory, the quartic invariant $\mathcal{I}_{4}(\mathcal{Q})$ of reducible models is usually named "discriminant" of the charge vector $\mathcal{Q}$ (see e.g. [86]).
} 


\begin{tabular}{|c|c|l|c|}
\hline$\left(\operatorname{sgn}\left(p^{2}\right), \operatorname{sgn}\left(Q^{2}\right)\right)$ & $\operatorname{sgn}\left(\mathcal{I}_{4}\left(\mathcal{Q}_{1}+\mathcal{Q}_{2}\right)\right)$ & constraint & $\begin{array}{c}\text { horizontal } \\
\text { orbit } \mathcal{O} / S O_{h}^{v}(2,2)\end{array}$ \\
\hline$(+,+)$ & + & $\mathcal{X}<-\frac{1}{2} \mathcal{W}^{2}$ & $\frac{S O(m, n)}{\mathrm{SO}(m-2, n)}$ \\
\hline$(+,+)$ & - & $\mathcal{X}>-\frac{1}{2} \mathcal{W}^{2}$ & $\frac{S O(m, n)}{\mathrm{SO}(m-1, n-1)}$ \\
\hline$(-,-)$ & + & $\mathcal{X}<-\frac{1}{2} \mathcal{W}^{2}$ & $\frac{S O(m, n)}{\mathrm{SO}(m, n-2)}$ \\
\hline$(-,-)$ & - & $\mathcal{X}>-\frac{1}{2} \mathcal{W}^{2}$ & $\frac{S O(m, n)}{\mathrm{SO}(m-1, n-1)}$ \\
\hline$(+,-)$ & - & $\mathcal{X}>0$ & $\frac{S O(m, n)}{\mathrm{SO}(m-1, n-1)}$ \\
\hline$(-,+)$ & - & $\mathcal{X}>0$ & $\frac{S O(m, n)}{\mathrm{SO}(m-1, n-1)}$ \\
\hline
\end{tabular}

Table 3. Two-centered charge configuration of the type (6.5) with $\operatorname{rank}\left(\mathcal{Q}_{1}, \mathcal{Q}_{2}\right)=(2,2)_{I I}$ in reducible symmetric models $(\mathcal{N}=2$ and $\mathcal{N}=4$ supergravity theories are obtained for $m=2$ and $m=6$, respectively). The cases with $\mathcal{I}_{4}\left(\mathcal{Q}_{1}+\mathcal{Q}_{2}\right)=0$ are not considered.

By considering both $\mathcal{Q}_{1}$ and $\mathcal{Q}_{2}$ of configuration (6.5) to be timelike or spacelike (this corresponds to $\operatorname{rank}\left(\mathcal{Q}_{1}, \mathcal{Q}_{2}\right)=(2,2)_{I I}$, given by subcase $I I$ of point 6 of section 4.1$)$ ), and recalling the analysis done in the second part od section 4 of [45], one obtains the case study reported in table 3 (in which the cases with $\mathcal{I}_{4}\left(\mathcal{Q}_{1}+\mathcal{Q}_{2}\right)=0$ have been disregarded), in which the sign of $\mathcal{I}_{4}\left(\mathcal{Q}_{1}+\mathcal{Q}_{2}\right)$ (second column) is equivalent to the constraint on $\mathcal{X}$ (third column), because it holds that

$$
\mathcal{I}_{4}\left(\mathcal{Q}_{1}+\mathcal{Q}_{2}\right)=\mathcal{I}_{4}(p, Q)=-\mathcal{W}^{2}-2 \mathcal{X}
$$

On the other hand, when $p^{\Lambda}$ and/or $Q_{\Lambda}$ of (6.5) are lightlike (or, equivalently, when $\mathcal{Q}_{1}$ and/or $\mathcal{Q}_{2}$ are rank 1 ; see eq. (6.7)), namely in the mutually non-local cases

$$
\begin{gathered}
p^{2}>0, Q^{2}=0: \operatorname{rank}=(2,1) ; \\
p^{2}=0, Q^{2}>0: \operatorname{rank}=(1,2) ; \\
p^{2}=0, Q^{2}=0: \operatorname{rank}=(1,1),
\end{gathered}
$$

$\mathcal{I}_{4}\left(\mathcal{Q}_{1}+\mathcal{Q}_{2}\right)$ is strictly negative (because $\mathcal{X}=0$ ), and it is given by eq. (6.2). Note that for the cases (6.10) $\mathcal{I}_{4}\left(\mathcal{Q}_{1}+\mathcal{Q}_{2}\right)=0$ is equivalent to mutually local centers.

Thus, the two-centered charge configurations $\left(\mathcal{Q}_{1}, \mathcal{Q}_{2}\right)$ of the heterotic type (6.5) given by the second, fourth, fifth and sixth line of table 3, and by eq. (6.10), are all characterized by the corresponding $\mathrm{BH}$ compound that, regarded as a single-centered (extremal) $\mathrm{BH}$, is "large" and non-BPS, with a negative quartic duality invariant $\mathcal{I}_{4}\left(\mathcal{Q}_{1}+\mathcal{Q}_{2}\right)$. These configurations are (the semiclassical limit, with real, continuous charges of) some of the cases recently analyzed by $\operatorname{Sen}^{8}{ }^{8}[68]$ in $\mathcal{N}=4, d=4$ supergravity; furthermore, the case $\operatorname{rank}\left(\mathcal{Q}_{1}, \mathcal{Q}_{2}\right)=(1,1)$ encompasses the $D 0+D 6$ configuration in the $\mathcal{N}=2$ stu model, which has been studied in [11]. Since both (6.2) and (6.9) are manifestly horizontalinvariant expression, the most general supporting two-centered configurations can be com-

\footnotetext{
${ }^{8}$ In the notation of [68], $m \equiv R$ and $n \equiv L$.
} 
puted by acting on the relevant case of the heterotic configuration (6.5) with a generic $\left[S O_{h}^{v}(2,2) \times S O(m, n)\right]$-transformation (also, recall (4.2)).

\section{Acknowledgments}

A. M. would like to thank Leron Borsten for interesting discussions, the Department of Theoretical Physics, University of Torino, for kind hospitality, and the ERC Advanced Grant no. 226455, "Supersymmetry, Quantum Gravity and Gauge Fields" (SUPERFIELDS) for financial support.

A.Y. would like to thank CERN Theory Division for kind hospitality.

The work of A. C., S. F. and A. Y. is supported by the ERC Advanced Grant no. 226455 SUPERFIELDS. S. F. is supported in part by DOE Grant DE-FG03-91ER40662.

Open Access. This article is distributed under the terms of the Creative Commons Attribution Noncommercial License which permits any noncommercial use, distribution, and reproduction in any medium, provided the original author(s) and source are credited.

\section{References}

[1] F. Denef, Supergravity flows and D-brane stability, JHEP 08 (2000) 050 [hep-th/0005049] [SPIRES].

[2] F. Denef, B.R. Greene and M. Raugas, Split attractor flows and the spectrum of BPS D-branes on the quintic, JHEP 05 (2001) 012 [hep-th/0101135] [SPIRES].

[3] B. Bates and F. Denef, Exact solutions for supersymmetric stationary black hole composites, hep-th/0304094 [SPIRES].

[4] F. Denef and G.W. Moore, Split states, entropy enigmas, holes and halos, hep-th/0702146 [SPIRES].

[5] F. Denef, D. Gaiotto, A. Strominger, D. Van den Bleeken and X. Yin, Black hole deconstruction, hep-th/0703252 [SPIRES].

[6] F. Denef and G.W. Moore, How many black holes fit on the head of a pin?, Int. J. Mod. Phys. D 17 (2008) 679 [arXiv: 0705.2564] [SPIRES].

[7] D. Gaiotto, W. Li and M. Padi, Non-Supersymmetric Attractor Flow in Symmetric Spaces, JHEP 12 (2007) 093 [arXiv:0710.1638] [SPIRES].

[8] M.C.N. Cheng and E.P. Verlinde, Wall Crossing, Discrete Attractor Flow and Borcherds Algebra, SIGMA 4 (2008) 068 [arXiv:0806.2337] [SPIRES].

[9] E.G. Gimon, F. Larsen and J. Simon, Constituent Model of Extremal non-BPS Black Holes, JHEP 07 (2009) 052 [arXiv: 0903.0719] [SPIRES].

[10] I. Bena, G. Dall'Agata, S. Giusto, C. Ruef and N.P. Warner, Non-BPS Black Rings and Black Holes in Taub-NUT, JHEP 06 (2009) 015 [arXiv:0902.4526] [SPIRES].

[11] A. Castro and J. Simon, Deconstructing the D0-D6 system, JHEP 05 (2009) 078 [arXiv:0903.5523] [SPIRES].

[12] J.R. David, On walls of marginal stability in $N=2$ string theories, JHEP 08 (2009) 054 [arXiv:0905.4115] [SPIRES]. 
[13] D. Gaiotto, G.W. Moore and A. Neitzke, Wall-crossing, Hitchin Systems and the WKB Approximation, arXiv:0907.3987 [SPIRES].

[14] P. Galli and J. Perz, Non-supersymmetric extremal multicenter black holes with superpotentials, JHEP 02 (2010) 102 [arXiv:0909.5185] [SPIRES].

[15] E. Andriyash, F. Denef, D.L. Jafferis and G.W. Moore, Wall-crossing from supersymmetric galaxies, arXiv: 1008.0030 [SPIRES].

[16] E. Andriyash, F. Denef, D.L. Jafferis and G.W. Moore, Bound state transformation walls, arXiv: 1008.3555 [SPIRES].

[17] J. Manschot, B. Pioline and A. Sen, Wall-Crossing from Boltzmann Black Hole Halos, arXiv: 1011.1258 [SPIRES].

[18] J. Manschot, B. Pioline and A. Sen, A fixed point formula for the index of multi-centered $N=2$ black holes, JHEP 05 (2011) 057 [arXiv: 1103.1887] [SPIRES].

[19] D. Gaiotto, G.W. Moore and A. Neitzke, Wall-Crossing in Coupled 2d-4d Systems, arXiv: 1103.2598 [SPIRES].

[20] S. Ferrara, E.G. Gimon and R. Kallosh, Magic supergravities, $N=8$ and black hole composites, Phys. Rev. D 74 (2006) 125018 [hep-th/0606211] [SPIRES].

[21] A. Sen, Walls of Marginal Stability and Dyon Spectrum in $N=4$ Supersymmetric String Theories, JHEP 05 (2007) 039 [hep-th/0702141] [SPIRES].

[22] A. Sen, Two Centered Black Holes and $N=4$ Dyon Spectrum, JHEP 09 (2007) 045 [arXiv:0705.3874] [SPIRES].

[23] A. Sen, N=8 Dyon Partition Function and Walls of Marginal Stability, JHEP 07 (2008) 118 [arXiv: 0803.1014] [SPIRES].

[24] A. Sen, Wall Crossing Formula for $N=4$ Dyons: A Macroscopic Derivation, JHEP 07 (2008) 078 [arXiv: 0803.3857] [SPIRES].

[25] G. Bossard and H. Nicolai, Multi-black holes from nilpotent Lie algebra orbits, Gen. Rel. Grav. 42 (2010) 509 [arXiv:0906.1987] [SPIRES].

[26] G. Bossard, The extremal black holes of $N=4$ supergravity from so $(8,2+n)$ nilpotent orbits, Gen. Rel. Grav. 42 (2010) 539 [arXiv:0906.1988] [SPIRES].

[27] G. Bossard, 1/8 BPS black hole composites, arXiv:1001.3157 [SPIRES].

[28] J. Rahmfeld, Extremal Black Holes as Bound States, Phys. Lett. B 372 (1996) 198 [hep-th/9512089] [SPIRES].

[29] M.J. Duff and J. Rahmfeld, Bound States of Black Holes and Other P-branes, Nucl. Phys. B 481 (1996) 332 [hep-th/9605085] [SPIRES].

[30] D. Gaiotto, A. Simons, A. Strominger and X. Yin, D0-branes in black hole attractors, hep-th/0412179 [SPIRES].

[31] A. Ritz, M.A. Shifman, A.I. Vainshtein and M.B. Voloshin, Marginal stability and the metamorphosis of BPS states, Phys. Rev. D 63 (2001) 065018 [hep-th/0006028] [SPIRES].

[32] P.S. Aspinwall, A. Maloney and A. Simons, Black hole entropy, marginal stability and mirror symmetry, JHEP 07 (2007) 034 [hep-th/0610033] [SPIRES].

[33] B. Pioline, Four ways across the wall, arXiv:1103.0261 [SPIRES]. 
[34] E. Cremmer and B. Julia, The $N=8$ Supergravity Theory. 1. The Lagrangian, Phys. Lett. B 80 (1978) 48 [SPIRES].

[35] E. Cremmer and B. Julia, The SO(8) Supergravity, Nucl. Phys. B 159 (1979) 141 [SPIRES].

[36] C.M. Hull and P.K. Townsend, Unity of superstring dualities, Nucl. Phys. B 438 (1995) 109 [hep-th/9410167] [SPIRES].

[37] S.W. Hawking, Gravitational radiation from colliding black holes, Phys. Rev. Lett. 26 (1971) 1344 [SPIRES].

[38] J.D. Bekenstein, Black holes and entropy, Phys. Rev. D 7 (1973) 2333 [SPIRES].

[39] R.L. Arnowitt, S. Deser and C.W. Misner, Canonical variables for general relativity, Phys. Rev. 117 (1960) 1595 [SPIRES].

[40] S. Ferrara and M. Günaydin, Orbits of exceptional groups, duality and BPS states in string theory, Int. J. Mod. Phys. A 13 (1998) 2075 [hep-th/9708025] [SPIRES].

[41] H. Lü, C.N. Pope and K.S. Stelle, Multiplet structures of BPS solitons, Class. Quant. Grav. 15 (1998) 537 [hep-th/9708109] [SPIRES].

[42] A. Ceresole, S. Ferrara and A. Marrani, Small $N=2$ Extremal Black Holes in Special Geometry, Phys. Lett. B 693 (2010) 366 [arXiv:1006.2007] [SPIRES].

[43] A. Marrani, Charge Orbits and Moduli Spaces of Black Hole Attractors, arXiv:1012.3559 [SPIRES].

[44] S. Ferrara, A. Marrani, E. Orazi, R. Stora and A. Yeranyan, Two-Center Black Holes Duality-Invariants for STU Model and its lower-rank Descendants, arXiv:1011.5864 [SPIRES].

[45] L. Andrianopoli, R. D'Auria, S. Ferrara, A. Marrani and M. Trigiante, Two-Centered Magical Charge Orbits, JHEP 04 (2011) 041 [arXiv:1101.3496] [SPIRES].

[46] P. Levay, Two-Center Black Holes, Qubits and Elliptic Curves, arXiv:1104.0144 [SPIRES].

[47] S. Ferrara and A. Marrani, Matrix Norms, BPS Bounds and Marginal Stability in $N=8$ Supergravity, JHEP 12 (2010) 038 [arXiv: 1009.3251] [SPIRES].

[48] A. Marrani, E. Orazi and F. Riccioni, Exceptional Reductions, J. Phys. A 44 (2011) 155207 [arXiv: 1012.5797] [SPIRES].

[49] E. Calabi and E. Vesentini, On Compact, Locally Symmetric Kähler Manifolds, Ann. Math. 71 (1960) 472.

[50] A. Ceresole, R. D'Auria, S. Ferrara and A. Van Proeyen, Duality transformations in supersymmetric Yang-Mills theories coupled to supergravity, Nucl. Phys. B 444 (1995) 92 [hep-th/9502072] [SPIRES].

[51] M. Cvetič and D. Youm, Dyonic BPS saturated black holes of heterotic string on a six torus, Phys. Rev. D 53 (1996) 584 [hep-th/9507090] [SPIRES].

[52] M.J. Duff, J.T. Liu and J. Rahmfeld, Four-dimensional string-string-string triality, Nucl. Phys. B 459 (1996) 125 [hep-th/9508094] [SPIRES].

[53] M. Cvetič and A.A. Tseytlin, General class of BPS saturated dyonic black holes as exact superstring solutions, Phys. Lett. B 366 (1996) 95 [hep-th/9510097] [SPIRES].

[54] M. Cvetič and A.A. Tseytlin, Solitonic strings and BPS saturated dyonic black holes, Phys. Rev. D 53 (1996) 5619 [hep-th/9512031] [SPIRES]. 
[55] S. Ferrara, A. Marrani and E. Orazi, Split Attractor Flow in $N=2$ Minimally Coupled Supergravity, Nucl. Phys. B 846 (2011) 512 [arXiv:1010.2280] [SPIRES].

[56] L. Andrianopoli, R. D'Auria, S. Ferrara and M. Trigiante, Fake Superpotential for Large and Small Extremal Black Holes, JHEP 08 (2010) 126 [arXiv: 1002.4340] [SPIRES].

[57] J.C. Ferrar, Strictly Regular Elements in Freudenthal Triple Systems, Trans. Amer. Math. Soc. 174 (1972) 313.

[58] S. Krutelevich, Jordan Algebras, Exceptional Groups, and Higher Composition Laws, J. Algebra 314 (2007) 924 [math/0411104].

[59] S. Ferrara and J.M. Maldacena, Branes, central charges and U-duality invariant BPS conditions, Class. Quant. Grav. 15 (1998) 749 [hep-th/9706097] [SPIRES].

[60] L. Borsten, D. Dahanayake, M.J. Duff and W. Rubens, Black holes admitting a Freudenthal dual, Phys. Rev. D 80 (2009) 026003 [arXiv:0903.5517] [SPIRES].

[61] R.B. Brown, Groups of Type E ${ }_{7}$, J. Reine Angew. Math. 236 (1969) 79.

[62] M. Günaydin, Lectures on Spectrum Generating Symmetries and U-duality in Supergravity, Extremal Black Holes, Quantum Attractors and Harmonic Superspace, arXiv:0908.0374 [SPIRES].

[63] L. Borsten et al., Observations on Integral and Continuous U-duality Orbits in $N=8$ Supergravity, Class. Quant. Grav. 27 (2010) 185003 [arXiv: 1002.4223] [SPIRES].

[64] J.F. Luciani, Coupling of O(2) Supergravity with Several Vector Multiplets, Nucl. Phys. B 132 (1978) 325 [SPIRES].

[65] L. Castellani et al., The complete $N=3$ matter coupled supergravity, Nucl. Phys. B 268 (1986) 317 [SPIRES].

[66] S. Bellucci, S. Ferrara, M. Günaydin and A. Marrani, Charge orbits of symmetric special geometries and attractors, Int. J. Mod. Phys. A 21 (2006) 5043 [hep-th/0606209] [SPIRES].

[67] S. Bellucci, A. Marrani, E. Orazi and A. Shcherbakov, Attractors with Vanishing Central Charge, Phys. Lett. B 655 (2007) 185 [arXiv:0707.2730] [SPIRES].

[68] A. Sen, Negative discriminant states in $N=4$ supersymmetric string theories, arXiv:1104.1498 [SPIRES].

[69] A. Galperin and O. Ogievetsky, Harmonic potentials for quaternionic symmetric $\sigma$-models, Phys. Lett. B 301 (1993) 67 [hep-th/9210153] [SPIRES].

[70] L. Borsten, D. Dahanayake, M.J. Duff, H. Ebrahim and W. Rubens, Black Holes, Qubits and Octonions, Phys. Rept. 471 (2009) 113 [arXiv:0809.4685] [SPIRES].

[71] L. Andrianopoli, R. D'Auria and S. Ferrara, U-invariants, black-hole entropy and fixed scalars, Phys. Lett. B 403 (1997) 12 [hep-th/9703156] [SPIRES].

[72] S. Ferrara, E.G. Gimon and R. Kallosh, Magic supergravities, $N=8$ and black hole composites, Phys. Rev. D 74 (2006) 125018 [hep-th/0606211] [SPIRES].

[73] L. Andrianopoli, R. D'Auria, S. Ferrara, P.A. Grassi and M. Trigiante, Exceptional $N=6$ and $N=2 A_{d} S_{4}$ Supergravity and Zero-Center Modules, JHEP 04 (2009) 074 [arXiv:0810.1214] [SPIRES].

[74] D. Roest and H. Samtleben, Twin Supergravities, Class. Quant. Grav. 26 (2009) 155001 [arXiv:0904.1344] [SPIRES]. 
[75] M.J. Duff and S. Ferrara, Generalized mirror symmetry and trace anomalies, Class. Quant. Grav. 28 (2011) 065005 [arXiv: 1009.4439] [SPIRES].

[76] M. Günaydin, G. Sierra and P.K. Townsend, Exceptional Supergravity Theories and the MAGIC Square, Phys. Lett. B 133 (1983) 72 [SPIRES].

[77] M. Günaydin, G. Sierra and P.K. Townsend, The Geometry of $N=2$ Maxwell-Einstein Supergravity and Jordan Algebras, Nucl. Phys. B 242 (1984) 244 [SPIRES].

[78] K. Behrndt, R. Kallosh, J. Rahmfeld, M. Shmakova and W.K. Wong, STU black holes and string triality, Phys. Rev. D 54 (1996) 6293 [hep-th/9608059] [SPIRES].

[79] R. D'Auria, S. Ferrara and M.A. Lledó, On central charges and Hamiltonians for 0-brane dynamics, Phys. Rev. D 60 (1999) 084007 [hep-th/9903089] [SPIRES].

[80] R. Slansky, Group Theory for Unified Model Building, Phys. Rept. 79 (1981) 1 [SPIRES].

[81] O. Shukuzawa, Explicit Classification of Orbits in Jordan Algebra and Freudenthal Vector Space over the Exceptional Lie Groups, Commun. Algebra 34 (2006) 197.

[82] K. Yokota, Exceptional Lie Groups, arXiv:0902.0431.

[83] L. Andrianopoli et al., $N=2$ supergravity and $N=2$ super Yang-Mills theory on general scalar manifolds: Symplectic covariance, gaugings and the momentum map, J. Geom. Phys. 23 (1997) 111 [hep-th/9605032] [SPIRES].

[84] P. Fré, A.S. Sorin and M. Trigiante, Integrability of Supergravity Black Holes and New Tensor Classifiers of Regular and Nilpotent Orbits, arXiv:1103.0848 [SPIRES].

[85] B.L. Cerchiai, S. Ferrara, A. Marrani and B. Zumino, Duality, Entropy and ADM Mass in Supergravity, Phys. Rev. D 79 (2009) 125010 [arXiv: 0902.3973] [SPIRES].

[86] G.W. Moore, Arithmetic and attractors, hep-th/9807087 [SPIRES]. 